\title{
Fractals in the nervous system: conceptual implications for theoretical neuroscience
}

\author{
Gerhard Werner* \\ Department of Biomedical Engineering, University of Texas at Austin, TX, USA
}

\section{Edited by:}

Dante R. Chialvo, Northwestern

University, USA

\section{Reviewed by:}

Henrik J. Jensen, Imperial College

London, UK

Matias Palva, University of Helsinki,

Finland

*Correspondence:

Gerhard Werner, Department of

Biomedical Engineering, University of

Texas at Austin, TX, USA.

e-mail: gwer1@mail.utexas.edu
This essay is presented with two principal objectives in mind: first, to document the prevalence of fractals at all levels of the nervous system, giving credence to the notion of their functional relevance; and second, to draw attention to the as yet still unresolved issues of the detailed relationships among power-law scaling, self-similarity, and self-organized criticality. As regards criticality, I will document that it has become a pivotal reference point in Neurodynamics. Furthermore, I will emphasize the not yet fully appreciated significance of allometric control processes. For dynamic fractals, I will assemble reasons for attributing to them the capacity to adapt task execution to contextual changes across a range of scales. The final Section consists of general reflections on the implications of the reviewed data, and identifies what appear to be issues of fundamental importance for future research in the rapidly evolving topic of this review.

\section{Keywords: criticality, phase transitions, self-organization, self-similarity, metastability, coordination dynamics}

\section{INTRODUCTION}

Fractals, introduced by Mandelbrot in 1977, are signals which display scale-invariant, self-similar behavior. Their signature is power-law scaling. In the spatial domain, fractals are self-similar geometric objects with features on an infinite number of scales. In the analysis of time series, they can constitute a hierarchy of temporal and spatial scales that may cover the wide range between coarse-scale long-term and high-frequency fine-scale fluctuations. The statistical analysis of Dynamical Fractals can provide access to understanding the dynamics of complex systems. Random Fractals refer to the participation of a stochastic process in their generation. If the relationship of the property under consideration is simple with respect to change of scale, the process is considered monofractal. The process can then be characterized by a single scaling exponent which is related to the fractal dimension or the spectral exponent of the process. It can be expressed as the Hurst exponent of the process (Mandelbrot and van Ness, 1968; Koutsoyiannis, 2002). However, in some instances the scaling behavior may not be adequately characterized by a single, stationary scaling exponent. In such cases, several scaling exponents may be required, each exponent locally pertaining to some portion of the data stream. Such multifractal signals are represented by the histogram of the Hoelder exponents, also known as the singularity spectrum (Muzy et al., 1993).

Power-law scaling and other manifestations of fractal and self-similar patterns in space and/or time can be identified at all levels of neural organization. With few exceptions, these observations remained largely islands in the otherwise rapidly advancing theoretical Neuroscience with different priorities. However, recent advances in methodology of measurement of fractal connectivity at higher levels of brain organization have led to a proliferation of new data. This now calls for integrating fractality with other insights into brain organization and complexity, notably in the light of the substantial evidence for the brain being a complex system in a regime of criticality, as understood in statistical physics (Chialvo, 2004; Chialvo et al., 2008; Kitzbichler et al., 2009; Fraiman et al., 2009; Werner, 2007b, 2009a,b). Like in other physiological systems manifesting fractal patterns (see for instance: Bassingthwaighte et al., 1994; West and Deering, 1995; Iannacone and Khokha, 1995; West, 2006) the question of ubiquity of power-law scaling needs to be addressed in relation to other features of brain organization. Similarly, is there a relation between fractal organization and the propensity for phase transitions of critical systems? Is there a bridge between coarse graining (including renormalization group transformation) and fractality? And, most importantly, can fractal properties be viewed as playing a role for the functional integration among different levels of neuronal organization as Andersen (2000) suggests in an article entitled "From Molecules to Mindfulness". Generalizing from a comprehensive theory of organization and interactions at the molecular level, Agnati et al. $(2004,2009)$ view the Central Nervous System as a nested network at all levels of organization, in the image of the Russian Matryoshka dolls: selfsimilar structures being embedded within one another. The authors elaborated this theory in great detail, with a "Fractal (self-similarity) Logic" operating on a set of identical rules which would govern the relation between successive levels of the nested system.

Although primarily concerned with chaotic dynamics in the Nervous System, King (1991) foresaw in an extensive review that "....fractal dynamics may be of functional utility in central nervous system cognitive processes". Gisiger's (2001) comprehensive overview of scale invariance in Biology provides the background of this review, as do the insights gained in Physics through the work of Wilson (1979) and Kadanoff (1990), among many others. In addition, Turcotte (1999) discussed at great length the relation between aspects of self-organized criticality and fractal scaling from the points of view of "avalanche" behavior and systematic properties of correlation length, specifically directing attention to inverse cascade and site-percolation models of the well-known forest fire 
paradigm. While none of the issues discussed in the following will receive a definitive answer, I will aim at an explicit formulation of the network of interrelated factors that constitute the territory in which new perspectives and potential solutions may lie.

With the agenda set forth in the foregoing, the organization of the presentation is as follows: I will first briefly review the neuroscience literature on fractals, organized by level of neuronal organization, from ion channels to cortical networks, and to psychological and behavioral functions. Criticality of brain states, Allometric control, and the origin of fractals by phase transitions of complex systems will be addressed in more detail. This will be followed by a brief overview of the essentials features of the theory of fractal generators, including random walk theory and fractional differential operators. Having laid out the background in this manner, I will consider relations between renormalization group transformation and fractals as having some potential bearing on the apparent ubiquity and universality of power-law scaling in neural structures and processes, and its relation to criticality. Finally, I will direct attention to the amazing consequence of self-similarity which assures the telescoping of different levels of structural and functional organization to constitute a fractal object or time series. This will lead me to posing the ultimate question: is there a process for unpacking interactions between different levels of the fractal object, responsive to circumstances and conditions, which eludes us entirely? If it existed, fractals would surely be a most extraordinary design principle for operational economy in complex systems.

\section{POWER-LAW SCALING IN NEURONAL STRUCTURES AND PROCESSES}

This section is intended to summarize essential aspects of fractal properties at each of the conventionally designated organizational levels, as the basis for conceptual consideration of relations across these levels. However, a word of caution is in order: the sketches of observational data in this section encompass a vast variety of biological substrates, conditions of observation, and methods of measurement. This heterogeneity imposes limits on generalizations, as do the differences of criteria for identifying fractal or self-similar features in the data. Potential pitfalls were discussed and illustrated in LaBarbera's (1989) useful (largely pedagogic) publication. More recently, Eke et al. (2002), Delignieres et al. (2006), and Clauset et al. (2009) set forth stringent criteria for design, collection, and interpretation of data for identifying and categorizing fractal properties. The latter authors are very specific in formulating a principled statistical framework, combining maximum likelihood fitting methods with goodness of fit tests; they demonstrate examples of data that had been conjectured to represent power-law fits, but did not withstand the rigor of their tests. The statistical properties of fractal time series as solutions of differential equations of fractal order or as responses of a fractional system are reviewed by Li (2010). Touboul and Destexhe (2009) also suggest that apparent power-law scaling may in some instances not be supported by more stringent statistical criteria. Of particular relevance to the topic of Section "The mesoscopic level" is their claim that experimentally observed power-law scaling must not considered proof of self-organized criticality, lest there be other supporting evidence available. The analysis, synthesis and estimation of fractal-rate stochastic point processes is reviewed and illustrated with examples by Thurner et al. (1997). Note also that the distinction between mono- and multifractal scaling is sometimes difficult to draw (Kadanoff et al., 1989).

Conceivably, some of the variations among the reports reviewed in subsequent sections may be attributable to procedural differences among studies; other reports may not meet the rigorous statistical criteria of Clauset et al. Nevertheless, I submit that the majority of experimental data on fractals in neural structures give collectively adequate reason for ascribing to them widespread functional significance. At least the results based on wavelet analysis appear immune to methodological criticism (see The macroscopic level).

\section{NEURONAL MORPHOLOGY}

In the foundational work "The fractal geometry of Nature", Mandelbrot (1977) wrote "it would be nice if neurons - he mentioned specifically Purkinje cells in the cerebellum - turned out to be fractal": Nature obliged abundantly as the following sample of findings with dendrites, neuron cell bodies and glia cells indicates. Studying the branching pattern of dendritic trees of retina neurons, Caserta et al. (1990) identify by box-counting fractal shapes with a fractal dimension of approximately 1.7 , which can be explained by a diffusion-limited aggregation model (Witten and Sander, 1981); but fractal dimension measured by different methods (for instance comparing box counting with cumulative mass method) gives appreciably different values (Caserta et al., 1995). A fractal structure was observed by Kniffki et al. (1994) for the branching dendrite patterns of thalamic neurons in Golgi impregnated specimens. In a separate series, a scaling relation for bifurcations within the dendrite trees was ascertained (Kniffki et al., 1993). Significant species differences in fractal dimensions of dendrite arborizations in dorsal horn spinal cord neurons (Milosevic et al., 2007) may be attributable to species differences in peripheral somesthetic sensibility (the dorsal horn neurons being the first receiving station of this type of afferent input). Fractal analysis also reveals a distinct differentiation of neuron types in the different laminae of the dorsal horn (Milosevic et al., 2005). Differences in regional connectivity and functional capacity among different regions in visual cortex pyramidal neurons are also associated with marked variation in the fractal dendrite branching structure (Zietsch and Elston, 2005). Fractal analyses provide a measure of space filling of dendrite arbors which, in a study by Jelinek and Elston (2001), differentiates in the macaque visual cortex the two known processing streams between primary and secondary visual area by differences in fractal properties. These investigators had undertaken a meticulous examination of criteria for 'quality control' in studies of this nature, from the stage of pre-processing of tissue specimens to comparative evaluation of methods for determining fractal dimension (Jelinek et al., 1995). Examining the connectivity repertoire of basal dendrite arbors of pyramidal neurons, Wen et al. (2009) determined a universal power-law scaling for dendrite length and radius, suggesting that the dendrite arbors are constructed by statistically similar processes; moreover, fragments of an arbor are statistically similar to the entire arbor, thus displaying self-similarity. These design features are thought to maximize functionality for a fixed dendrite cost.

Additional evidence comes from digital image analysis which enabled Smith et al. (1989) to determine the fractal dimension of neuron contours. Results obtained with conventional methods of 
scaling analysis are corroborated by Wavelet Packet fractal analysis (Jones and Jelinek, 2001). Multifractals were identified for cortical pyramidal cells while, in comparison, neurons of synRas transgenic mice display less complex arborization patterns (Schierwagen, 2008.). Shape complexity of neurons and elements of microglia in human brain can be classified over a range for fractal dimensions which is different for normal and pathological brains (Karperien and Jelinek, 2008). The sequence of developmental stages of oligodendrocytes, tracked the basis of their immunoreactivity, parallels changes in fractal dimension (Bernard et al., 2001). Fractal analysis of cell ramification and space filling patterns differentiate microglia cells into two categories, depending on whether their fractal dimension did, or did not increase after brain injury (Soltys et al., 2001).

The scaling law for the cortical magnification factor in primate visual cortex is an illustrative example with functional significance: as is well known, the part of the visual scene corresponding to the eye center is represented densely at the cortex, becoming progressively sparser towards the periphery. It turns out that the scaling law for the sampling density away from area centralis is a powerfunction which assures locating a peripheral target in the shortest time (Koulakov, 2010). As brain size increases, the cortex thickens only slightly, but the degree of sulcal convolution increases dramatically, indicating that human cortices are not simply scaled versions of one another (Im et al., 2008). Changizi (2003) infers several organizing principles of Neocortex from scaling relations among its components: e.g. scaling of diameters of neural structures would predispose for efficient transport through neuron arborizations; economical wiring reflects well-connectedness within given volumes of neural tissue. These relations are viewed to represent a universal law for scaling that applies to hierarchical complexity and combinatorial systems, generally (Changizi, 2001b; see also: West et al., 1997b; West and Brown, 2004).

As one among several instances of scaling in the cerebral cortex, Changizi (2001a) also shows that axon cross sectional area increases in Phylogeny with brain size, presumably compensating increase of conduction distances with increase of conduction velocity (see Symbol processing and fractals). A synthesis of comparative Neuroanatomy with biophysics leads Harrison et al. (2002) to conclude that scaling trends in morphological specializations at the cellular level may constitute functional adaptations. One of the examples in support of their thesis is the role of component scaling for managing the conflicting developmental trends of increasing brain size and surface folding on the one hand, and the requirement for optimizing energy requirements and processing speed, on the other. The role of scaling relations for brain growth becomes evident when its scaling relation is disrupted by preterm birth in a dose-dependent, sexually dimorphic fashion; it directly parallels the incidence of neuro-developmental impairments in preterm infants (Kapellou et al., 2006).

Taken together, the observations surveyed in the foregoing two paragraphs suggest that fractal dimension of neuronal and glia elements bear some relations to developmental, functional, and pathological conditions of neural tissue. This warrants a few conceptual considerations: Bieberich (2002) attaches neural-computational significance to the self-similarity of dendritic branching as a platform for economical information compression and recursive algorithms. On the same self-similarity principle, Pellionisz (1989) envisages a fractal growth model of dendritic arbors by iterated code repetition as process for global construction of fractals (see for instance: Barnsley and Demko, 1985): the essential underlying theme is to both reduce complexity of generating, and at the same time conserving the full richness of the dendrite arbor. I will expand on this principle in later section of this essay. Among the not yet explored implications of dendrite fractal arborizations are the effect they may induce on the dynamics of processes and critical phenomena in dendrite spines for which they are a supporting platform: In Statistical Physics, such effects obtain when the neighborhood relations among interacting elements [for instance: Ising spins or coupled maps (Cosenza and Kapral, 1992)] are themselves provided by a self-similar fractal lattices, such as the Sierpinski Gasket (Gefen et al., 1980), rather than an Euclidean geometric base.

In an extension of fractal analysis to features of complex neural structures, Zhang (2006) determined the Magnetic Resonance image-based fractal dimension of white matter of the human brain. This method was shown to accurately quantify white matter structural complexity in three dimensions, and detect age-related degenerative changes. Tractography based on Diffusion Tensor Imaging enabled Katsaloulis and Verganelakis (2009) to determine fractal dimension, self-similarity, and lacunarity of neuron tracts in the human brain. The lacunarity analysis is understood to indicate the distribution of fractal neuron tracts of different length scales, as evidence of connections between different neuron ensembles. Another extraordinary technical advance made it possible to determine the fractal properties of receptor density and distribution in human brain, using Positron Emission Tomography (PET) and Single-photon Emission Tomography (SPET) (Kuikka and Tiihonen, 1998).

\section{THE PERIPHERAL NERVOUS SYSTEM: ION CHANNELS, POINT PROCESS ANALYSIS OF ACTIVITY IN PERIPHERAL NERVES AND INDIVIDUAL NEURONS Ion channels}

Turning to primarily functional aspects of fractality in neural systems, attention focuses in this section on temporal aspects of ion channel gating and its relation to time series of neuronal discharge patterns. The following collage of data obtained with different experimental conditions as well as modeling studies consistently supports the dominant presence of fractal features in the functional manifestations at the levels under consideration. The kinetics of Ion transport across neuronal membranes occurs, in part, via ion channels. Application of the Patch Clamp Technique made it possible to follow the time course of channel opening and closing precisely. Typically, the rate of channel opening and closing opening fluctuates, changing at times suddenly from periods of great to periods of slow activity. This pattern served as clue to surmise an underlying fractal process with infinite variance. On this basis Liebovitch et al. $(1987,2001)$ asked how the switching probabilities at one time scale of observation are related to those at another time scale. It turned out that these probabilities (defined as effective kinetic rate) at a given time scale are characterized by fractal scaling, and that effective kinetic rates for different time scales of observation display self-similarity: there are bursts within bursts of openings and closings. The suggestion is that energy barriers 
in stochastically switching protein conformational states are the underlying mechanism (for a detailed account, see Chapter 8 in Bassingthwaighte et al., 1994). A different version that also accounts for the power-law relationship of ion channel gating kinetics assumes that ion channel proteins have a very large number of states, all of similar energy, making the gating process more akin to a diffusion (Millhauser et al., 1988). Recent theoretical modeling defined more precisely the conditions that give rise to the powerlaw distributions in relation to the activation barriers, compatible with the known Physics of proteins (Goychuk and Hanggi, 2002). Roncaglia et al. (1994) developed on theoretical grounds a stringent criterion for ascertaining the validity of the fractal theory by evaluating the experimental distribution of channel closing times in terms of the Hurst phenomenon. A few years thereafter, Varanda et al. (2000) delivered the evidence for Ca-activated K channels in the form of long-term correlations of open and closed dwell times, expressed as Hurst coefficients of the order of 0.6 , which alternative Markovian models failed to satisfy.

Before proceeding to discuss the implication of channel kinetics for the patterning of trains of neuron spikes, a brief remark on the fractal activity at the site of neural impulse transmission at the neuromuscular junction. As is well known from the work of DelCastillo and Katz (1954), the neural transmitter substance acetylcholine is released from the nerve terminal in small packages: the miniature end potentials (MEPP) are considered manifestations of the exocytosis of humoral transmitters. In departure from initial textbook accounts of the MEPP release reflecting a set of homogeneous stationary Bernoulli trials, Perkel and Feldman (1979) categorically reject a purely binomial model of (quantal) transmitter release. For the frog neuromuscular junction, Rotshenker and Rahamimoff (1970) could show that excocytosis can exhibit correlations (memory) extending over periods of seconds, suggesting self-similar characteristics. When sampled over prolonged periods, Lowen et al. (1997) collected conclusive data at the neuromuscular junction and synapses in hippocampal tissue culture that frequency and amplitudes of MEPP's display fractal scaling. Takeda et al. (1999) also reported comparable findings for the vertebral neuromuscular junction. The detailed analysis of quantitative features of the recorded data led Lowen et al. (1997) to conclude that traditional renewal models of vesicular exocytosis as a memoryless stochastic process are entirely inadequate for representing many of its salient features. Instead, their recommendation is that a new class of models should be considered that relies on fractal-rate stochastic point processes: fractal-rate activity represents a kind of memory in that occurrence of an event at a given point in time increases the likelihood of another event to occur at a later point in time, with that likelihood persisting for some time.

\section{Point process analysis in peripheral nerves and neurons}

In this section, neuron discharge trains are viewed as mathematical objects, belonging to the class of point processes (Thurner et al., 1997; Lowen and Teich, 2005): events occurring at a point in time or space. Werner and Mountcastle $(1963,1964)$ determined scaling of neural responses in primary cutaneous afferent nerve fibers with the magnitude of mechanical stimuli applied to receptors. The implications of their findings in Psychophysics will be taken up in Section "Psychological and behavioral processes". Adaptation in neural structures serves to extend their dynamic range. The significance of this function is discussed in Section "Linking across many scales of space and time".

The statistics of action potential trains recorded from single neurons in the cochlear nucleus of anaesthetized cats formed the basis of a mathematical analysis by Gerstein and Mandelbrot (1964). The principal result was that a random walk model towards an absorbing and a reflecting barrier can account for a wide range of fractal neuronal activity patterns, assuming no more than the known physiological mechanisms of a threshold for membrane depolarization, and the summation of excitatory and inhibitory postsynaptic potentials. Except for a Thesis by Johannesma in 1969, it took almost 20 years of hegemony of Poisson and Gaussian distributions until fractal approaches to spike train statistics were resumed: this time by Wise (1981) in a study of spike interval distributions of data that had been recorded primarily by Bloom (1969) in the cerebral cortex, and in respiratory neurons recorded by Smolders and Folgering (1977). Wise found that plots of the spike interval histograms on log-log scales showed negative powers on time with long tails, which he attributed to the neuron membrane potential undergoing a random walk while the firing threshold fluctuates. Re-working some of Wise's data, West and Deering (1994) identified fractal (hyperbolic) spike interval distributions. Taking an entirely different approach to conceptualizing irregular behavior in neuron spike trains led Shahverdian and Apkarian (1999) to discuss self-affinity, power-Law dependence, and computational complexity of spike trains in terms of a multidimensional Cantor space with zero Lebesgue measure as attractor.

The turning point in the history of identifying fractal neuronal firing is associated with the work of Teich and Lowen, beginning in the early 1980s (Chapter 22, in McKenna, 1992) with invalidating the then prevalent notion of Poisson point processes. More recently, the shortcoming of Poisson spike interval statistics was also pointed out by Kass and Ventura (2001) and by van Vreeswijk (2001) who criticized experimental (Richmond et al., 1990) and theoretical (Ohlshausen and Field, 1997) reports for unwarrantedly assuming either Poisson neurons or rate based neurons with rate independent Gaussian noise; instead he considered a renewal model as biologically more plausible.

Teich and Lowen's essential realization was that determining long-time correlations in spike trains requires sample sizes to be appreciably larger than conventionally used. On this basis, Teich et al. (1990) identified the following essential features of the time series of neural spikes recorded from cat auditory nerve fibers and the lateral superior olivary nucleus: discharge rates determined with different averaging times can exhibit self-similarity; the varianceto-mean ratio of spike number increases with sufficiently large counting time in a fractional power-law fashion, with the exponent in the power-law varying with the stimulus level. With these data in hand, Lowen and Teich (1993) suggested that the fractal action potential patterning in auditory nerve may be related to fractal activity in the ion channels of the sensory organs feeding into the auditory nerve: that is, the hair cells in the cochlea. This idea required to show that ion channel gating and neuronal spiking patterns are indeed causally related. Lowen et al. (1999) succeeded with demonstrating this causal dependence in computational models, thus adding for the special case of the cochlear hair cells some 
credence to their proposal that gating patterns in sensory organ ion channels can affect discharge patterns in the sensory nerve tracts they feed. In an elegant experimental design, Teich et al. (1997) not only ascertained a power-function for the activity in retina ganglion cells and neurons in the lateral geniculate body when studied independently, but also succeeded with recording from synaptically connected pairs of retina ganglion cells and geniculate neurons. In this situation, fractal exponents for retina and target neurons in the lateral geniculate body were nearly identical. This was interpreted to mean that fractal behavior is either transmitted across synapses, or has a common origin for the synaptically connected pre- and postsynaptic structure. On the other hand, fractal activity of medullary sympathetic premotor and the synaptically connected pregangionic sympathetic neurons is apparently generated independently (Orer et al., 2003).

More support for the notion that ion channel properties play an important role for determining neuron performance comes from demonstrating a kind of memory mechanism for traces of prior activity in voltage-gated $\mathrm{Na}$ channels (Toib et al., 1998): time constants of channel recovery stand in a power-function relation to duration of prior activation. The question of primary interest is of course how the dynamics of ion channels relates to the functional characteristics of a whole neuron. Gilboa et al. (2005) addressed this question in a computational model of an ensemble of ion channels. In analogy to a "real" neuron, this model neuron exhibits various dynamics at different time scales: a power-law recovery time scale after stimulation, temporal modulation of discharge pattern during maintained stimulation, and the dependence of adaptation to a stimulus step on the duration of the priming stimulus. The suggestive implication is that the ensemble of ion channels can exhibit properties on many scales, comparable to "real" neurons, thus supporting the notion that the "macroscopic behavior" of the "real" neuron is, in fact, the result of cooperative fractal channel kinetics.

In addition to the studies cited in foregoing paragraphs, there are numerous reports documenting fractal-rate behavior in single neuronal point processes. However, these data were generally obtained for examining spike trains for encoding stimulus properties, and they are quite heterogeneous as regards species, neural structure examined, use of anesthetics and experimental conditions. Although this imposes serious limitations on drawing inferences on general principles, I select here a few studies which applied several of the commonly agreed upon and typical indicators of fractal properties, such as self similarity of firing rate with different averaging time, increase of spike number variance-to-mean ratio with counting time, and power-law scaling relating the variable of interest to the resolution of measurement. In a series of publications, Grüneis et al. (1993) reported fractal properties in spike trains recorded under various conditions including REM sleep of cats. In visual cortical areas of cats and macaques, Baddeley et al. (1997) observed consistently non-Poisson spike train statistics, with some displaying self-similarity. Other neural structures examined included medullary sympathetic neurons (Lewis et al., 2001) and dorsal horn of the spinal cord (Salvadori and Biella, 1994). A common feature of these and other reports not cited here, was the lack of agreement on a consistent mathematical model that would satisfactorily describe the fractal process underlying the experimental data. In a study of retina ganglion cells, Teich and Saleh (1981) suggest a shot-noise driven self-exciting point process; in a later study of the same experimental object, Teich found a modulated gammarenewal process satisfactory, while Grüneis et al. (1993) favored a clustering Poisson process. Electronic shot-noise was recently re-introduced by Milstein et al. (2009) for modeling power-law scaling within the power spectrum of LFPs. Mandelbrot and van Ness (1968) considered Fractal Brownian motion as candidate. Clearly, the goal of determining whether a common principle governing spike train variability could be identified, and if not then for what reason, eluded this group of investigators.

Without examining specifically for manifestations of fractality, a number of investigators attempted statistical characterization of neural point processes, primarily motivated to reconcile irregularity of spike trains with their presumptive function as "code" of neural signals. But recall that Harris (2005) attributes irregularity of spike train discharges to cell assembly organization. In various modifications, the general approach chosen by Sakai et al. (1999), Cateau and Reyes (2006), and Feng and Zhang (2001) consisted in designing model neurons to generate spike trains whose statistics would match that of "real" neurons recorded in animal experiments. Shinomoto et al. (2003) recorded spike sequences from different cortical areas in awake macaques which they classified phenomenologically into different groups. Salinas and Sejnowski (2002) and Stevens and Zador (1998) assigned the principal source of discharge variability to correlations in the input feeding the examined neuron. None of these results warranted the allocation of observed or simulated spike train data to one of the probability distributions in the conventional repertoire of statistics, but Maimon and Assad (2009) at least excluded Poisson - like randomness from being a universal feature of spike time distributions in primate parietal cortex. In an exquisitely elegant experiment, Evarts (1967) followed the changes of inter-spike interval (ISI) histograms in premotor cortex pyramidal neurons in wakefulness, sleep, and the phase of sleep associated with low-voltage fast EEG. Regrettably, his characterization of the ISI histograms is only limited to rejecting Poisson distributions, but inspecting the histograms displayed in Figure 12 of his publication arouses one's suspicion of a long-tail distribution for sleep activity.

In a notable and very extended comparison of cortical neuron discharges in alert macaques, Shadlen and Newsome (1998) attributed to single neurons the ability to perform simple algebraic operations resembling averaging by combining inputs from several sources, but they cautiously concluded that irregularity of the ISI distribution precludes them from reflecting information about the actual temporal structure of the synaptic input. They rejected random walk models of the kind applied by Gerstein and Mandelbrot as inadequate for capturing the statistical features of spike interval distributions, and found Poisson and various renewal processes likewise failing to yield satisfactory and consistent correspondence with recorded data.

If there is one conclusion that can be drawn from the extant data on the statistics of spike interval distributions, then it is that demonstrating fractal properties in spike trains requires carefully selected conditions. Multiple convergences from incoming pathways appears to obscure characteristic statistical properties of discharges in the recipient neurons. Thus, a neuron's intrinsic 
connection pattern carries the burden of discharge variability. This view is reminiscent of Harris's (2005) view that spike discharge variability may be a signature of cell assembly organization. This is perhaps also the source of futility of assigning any information bearing capacity to discharge patterns of individual neurons in labeled-line information transmission (see for instance: Werner, 2007a). On the other hand, the more direct a neuron's connection pattern to peripheral sensors is, the more distinctly are fractal discharge properties demonstrable. But the opposite also seems to be the case when neurons are embedded in a network, as the observations of El Boustani et al. (2009) in Section "The mesoscopic level” show. The place to look for consistent fractal properties is apparently the macroscopic, global level of brain organization (see The mesoscopic level). Whether and how the mesoscopic level of the next Section bridges the gap is the subject of the next section.

\section{THE MESOSCOPIC LEVEL OF ORGANIZATION}

Despite their relative simplicity, in vitro cultured neuronal networks are here viewed as mesoscopic in the sense of representing neuron ensembles which exhibit rich spontaneous dynamical activity in the form of periodic bursting (Robinson et al., 1993; Nakanishi and Kukita, 1998; van Pelt et al., 2004; earlier references are cited in: Beggs and Plenz, 2003, 2004). At superficial inspection, brief bursts of activity lasting tens of milliseconds are separated by quiescence lasting up to several seconds (Corner et al., 2002; Tateno et al., 2002). The spontaneous emergence of patterns in the discharge trains was also noted by Giugliano et al. (2004) and replicated in computational models. In extension of earlier work that led to identification of scale invariant Levy distributions and long-range correlations in cultured neuronal networks (Segev et al., 2002; see Processes that generate power-law distributions), Segev et al. (2004) attributed the activity bursts to be associated with the formation of statistically distinguishable subgroups of neurons, each with its own distinct pattern of interneuronal spatiotemporal correlations. Wagenaar et al. (2006a) emphasized the extremely rich repertoire of bursting patterns during the development of cortical cultures. The cultured cortical networks spontaneously generated a hierarchical structure of periodic activity with a stereotyped population-wide spatiotemporal structure. These recurring patterns (called by the authors 'superburst') converged periodically to a dynamic attractor orbit, and were taken to imply large-scale self-organization of neurons in vitro, refuting the commonly held view of having random connectivity (Wagenaar et al., 2006b). The recorded data of these authors are available for distribution to interested investigators. Departing from the practice of focusing on spontaneous activity, Breskin et al. (2006) explored the propagation of stimulus evoked activity in neuron cultures. A graph theoretic analysis of their data attributed the dynamic evolution of the network connectivity to a percolation transition with power-law characteristics, while the degree distribution of the grown network failed to meet power-law criteria.

Working with mature organotypical cultures and acute slices of rat cortex, Beggs and Plenz $(2003,2004)$ concluded that the cascades of propagating neuron discharges they observed were indicative of the neural culture being in a state of self-organized criticality. The importance of this claim, and a recent disputes of its validity (see below), warrant close attention to methodological details: Beggs and Plenz based their analysis on recording spontaneously appearing negative local field potentials (NLFP), apparently occurring preferentially in cortical layers 2/3 (Gireesh and Plenz, 2008). The peaks of NLFP were considered indicative of synchronized population discharges occurring near the recording electrode tip (Plenz and Aertsen, 1996), on the rational that bursts of multiunit activity are more likely to generate large NLFP's than are single neuron discharges (Plenz and Thiagarajan, 2007). Accordingly, brief bursts of synchronized action potentials were the units of analysis in their experiments. Intracranially recorded LFP in human brain show ubiquitous powerlaw scaling within the power spectrum (Milstein et al., 2009).

With the alternation between brief burst of NLFP activity and quiescent periods remaining stable with a high degree of temporal precision over periods of many hours, Beggs and Plenz set out to examine the idea that these cascades of neural activity may constitute a special mode of network activity, possibly of the character of "avalanches", indicative of SOC (Bak et al., 1987). They had specifically in mind the kind of self-organizing branching process discussed by Zapperi et al. (1995) and de Carvalho and Prado (2000). To examine this idea required determining the statistical properties of the observed activity patterns. For carrying out this analysis, they defined the spatial pattern of signal-carrying electrodes during one time bin as a frame; a sequence of consecutively active frames, preceded and ended by a blank period was called an avalanche. The NLFP did not propagate in the network in a spatially contiguous manner, thus excluding wave-like propagation. With these definitions and precautions in place, distributions of avalanche size and lifetimes were found to scale in cultures and acute cortex slices with a power-law exponent $-3 / 2$, this value being resilient to various choices of scales (Plenz and Thiagarajan, 2007). The branching parameter was determined as sigma $=1.04$. Being statistically indistinguishable from the ideal value of 1.0, it signifies a critical state in the sense that activity starting at one electrode would initiate activity in one other electrode, on the average, keeping the network at the edge of criticality (Harris, 1989). Critical branching also maximizes the number of metastable states in living neural networks (Haldeman and Beggs, 2005). This complements the evidence for fractal properties and supports the validity of the working hypothesis Beggs and Plenz started out with: to view avalanches as manifestations of the collective critical dynamics of SOC. More recently, fractal scaling of patterned neural activity was reported to also occur in cultivated neurons of leech ganglia and rat hippocampus (Mazzoni et al., 2007); and Pasquale et al. (2008) describe avalanches in dissociated neuronal cultures of cortex from embryonic rats.

Avalanches were subsequently also studied in superficial layers of rat prefrontal cortex (Stewart and Plenz, 2006) and during development of cortical layer 2/3 where they display nested theta- and beta/gamma oscillations (Gireesh and Plenz, 2008). The theory of critical states predicts (see Significance of Brain Criticality) and experiments confirm that neuronal avalanches display a maximized dynamic range of responses to stimuli (Shew et al., 2009). Functional architecture of avalanches conformed to Small World Topology (Pajevic and Plenz, 2009). Comparing NLFP activity in vitro cortex preparations with in vivo activity of awake macaque monkey cortex, Petermann et al. (2009) established that high fidelity propagation of local synchronized scale-invariant activity patterns is a robust and universal feature of cortex in awake monkeys. Furthermore, large amplitude negative field potentials (like 
those constituting the avalanches) spread in a cascade-like fashion through the cortical network without distortion, much like action potentials: Thiagarajan et al. (2010) described these stereotypical waveforms as "coherence potentials". They occur often in rapid succession as a stream of dynamical associations, suggesting the switching of the cortical network from one dynamical state to another.

The reason for viewing neuronal avalanches as manifestation of self-organized criticality was based on their fractal scaling properties for size and duration. Here just a brief reminder of the amply documented 'family resemblance' of fractality and SOC, to which the publications of Grinstein (1995), Chen et al. (1995), Paczuki et al. (1996), Tebbens and Burroughs (2003), and Cessac (2004) speak, as do the model computations of Papa and da Silva (1997), da Silva et al. (1998), de Arcangelis et al. (2006), and Levina et al. (2007). However, despite the 'avalanche' of research on mechanisms leading to scale invariance, there exist questions about the necessary conditions for establishing the self-organized critical state (Kinouchi and Prado, 1999). A non-conserving critical branching model was proposed by Juanico et al. (2007) to demonstrate that SOC can occur in mean-field sand piles, provided the branching process is coupled to a background activity of spontaneous switching between refractoriness and quiescence among system components; in the stationary state, this system can undergo a transition from a subcritical to a critical state. In an elaborate recent study, Bonachela and Munoz (2009) claim that non-conserving (dissipative) dynamics does not lead to bona fide criticality. Accordingly, non-conserving systems are in their view not truly critical models. Instead, non-conserving systems (such as the brain) would just hover around a critical point (presumably after some form of fine-tuning) with broadly distributed fluctuations which do not disappear at the thermodynamic limit. Such systems could be fluctuating in the vicinity of the critical point, but not at it. The authors call this condition "self-organized quasi-criticality". Whether this is the last word in the long standing debate of conditions for criticality in SOC remains yet to be seen. The earlier work of Kinouchi and Prado (l.c.) seems to offer a compromise in the form of a distinction between "true" criticality occurring at a special point, and a region of almost critical behavior. A novel aspect is presented by the neural model of Levina et al. (2009): it attains criticality in an Extended region of the parameter space, where, it can display Classical first- and second-order phase transitions as well as an SOC phase, depending on regulatory mechanisms at the level of synaptic connections. In any case, however, the dynamic regime leading to avalanche behavior may be considered a manifestation of a generic and persistent critical state, similar to that postulated by Coordination Dynamics: this will be pursued in Section "Fractals and Criticality of Brain States".

Conditions for universality of $1 / \mathrm{f}$ scaling in dissipative selforganized criticality models were established by De Los Rios and Zhang (1999). Models predicting avalanches of neural activity include the work of Herz and Hopfield (1995) and were noted by Eurich et al. (2002) in a network of globally coupled nonlinear threshold elements. Models of neural networks of non-leaky integrate-and-fire neurons exhibit over a wide range of connectivity patterns power-law avalanches with an exponent closely approximating that reported by Beggs and Plenz for tissue cultures (Levina et al., 2007). In a comment to this paper, Beggs (2007) gives a lucid account of how neural networks could self-organize to operate at criticality. Critical avalanche networks can be computationally constructed by simple network growth models (Abbott and Rohrkemper, 2007). An exponent of the experimentally determined value -1.5 of avalanche size and lifetime scaling is predicted by the neural field theory of Buice and Cowan (2007).

A field-theoretic approach was also investigated by Freeman (2005): analysis of high-resolution electroencephalograms of rabbits revealed neural fields in the form of spatial patterns in amplitude and phase modulation of gamma and beta carrier waves which distinguished positive and negative conditioning stimuli. The goal of applying field theory was in these experiments to model states and state transitions as large-scale spatial patterns of neural activity for quick changes in adjustment to different behavioral situations. The cortical states were viewed as "wave packets", resembling frames in motion picture, stabilized in a scale-free state of self-organized criticality. Recall, however, the reservations raised by Touboul and Destexhe (2009) that substantiating interdependence of fractality and self-organization requires additional evidence. In an entirely different context (namely fossil extinction), Newman (1996) shows that fractality need not be a unique indicator of SOC and criticality since an alternative simple model can account for the empirical power-law relation (for an extended discussion of this, see Processes that generate power-law distributions and Fractals in Action).

Seeking to strengthen the conjecture of self-organized criticality of avalanches, Plenz and Chialvo (2010) acquired experimental evidence that the neural avalanches in superficial layers of cortex exhibit five additional quantitative aspects of their dynamics which are consistent with critical dynamics. These were: separation of time scales between triggering and the avalanching event itself; stationarity of size distribution; temporal clustering prior to and following large avalanches, resembling Omori's law; power-law decay for avalanche size in the wake of preceding large avalanches; and a fractal dimension for scaling spatial spread. The importance of these results lies first, in affirming evidence for avalanches displaying robust critical behavior; and second, in suggesting that their scale-invariant (fractal) properties do in fact reflect cortical networks being in a state of criticality. This is also supported by the observation that the exponents of simulated branching processes at near-critical branching are similar to scaling exponents characterizing oscillations in the MEG recorded alpha frequency band of humans at rest (Poli et al., 2008).

It may be revealing to contrast the failure of consistently finding fractal activity patterns in individually sampled neurons (other than those receiving relatively direct input, see Point process analysis in peripheral nerves and neurons) with the abundance of fractal patterns of (mesoscopic) neuron ensembles. It raises the question whether the origin of the latter may be a matter of assembly organization: note that in the records of neuron cultures, it is the concurrent activity of interconnected neurons sampled by different electrodes that forms the fractal pattern; this is of course quite different from the sampling of neurons in point process analysis, guided by chance encounters of a microelectrode with one active neuron at the time. The puzzle posed at the end of Section "Point process analysis in peripheral nerves and neurons" thus finds perhaps its resolution in network architecture: Teramae and Fukai (2007) describe a model that shows how the fractal property of a few individual neurons can turn into an organized communal property 
of an ensemble. This lesson can also be learned from models of dynamic pattern formation in neuron populations, forming fractal power-spectra and power-law pulse distribution (Usher and Stemmler, 1995). Similarly, network amplification of local fluctuations causes fractal firing patterns and oscillatory field potentials in two-dimensional models of leaky Integrate-and-Fire neurons; feedback connectivity of local excitation and surround inhibition being the essential prerequisites (Usher et al., 1994).

Bedard et al. (2006b) accept existing claims for 1/f scaling of global variables of neural activity (e.g. EEG: Freeman et al. (2003); EMG: Novikov et al. (1997); see The mesoscopic level), and acknowledge them as evidence for self-organized critical states with power-law distributions, much as the models of de Arcangelis et al. (2006) and Levina et al. (2007) suggest. They also accept the validity of claims for fractality discussed in Section "Point process analysis in peripheral nerves and neurons" for various point process analyses. But they contest the legitimacy of generalizing from these disparate sources of data. The connection between $1 / \mathrm{f}$ frequency scaling of global variables and critical states of neural activity is, in their mind, far from firmly established. Moreover, they emphasize that the association of $1 / \mathrm{f}$ spectra with criticality may not be obligatory (for a review: see Newman, 1996; Giesinger, 2001).

Having raised this warning flag, Bedard et al. (2006a) not only confirmed in their own investigations the presence of $1 / \mathrm{f}$ frequency scaling in EEG of cat parietal cortex (in absence of anesthesia), but showed in addition 1/f frequency scaling in bipolar records of local field potentials (LFP). That bipolar LFP recordings sample relatively localized populations of neurons was shown by Destexhe et al. (1999). They then turned their attention to investigating whether this $1 / \mathrm{f}$ scaling reflects self-organized critical states with the result that avalanche size distributions in their experiments did not follow power-law scaling, nor did inter-spike interval distributions of concurrently recorded single neuron activity show 1/f scaling; rather, the distributions were consistent with a Poisson process. Accordingly, Bedard et al. reject the evidence for critical state dynamics. Instead, they proposed a model that would account for 1/f frequency scaling without being associated with critical states. Their model shows that the observed 1/f scaling can indeed be produced by a band pass filtering effect of extracellular media. This means that extracellular field potentials (such as LFP) can show power-law scaling while the underlying neuronal activity per se need not be critical. El Boustani et al. (2007) found in experimental data and models of cortical "activated" states also evidence for Poisson spike distributions, and absence of avalanche dynamics. The irregular states of cortical networks are thought to stem from a very high-dimensional deterministic chaos. However, as alternative, it is worth recalling that Harris (2005) views spike train irregularity as one of the signatures of cell assembly organization.

Investigating further the discrepancy between the Beggs-Plenz and the Bedard et al. observations, Touboul and Destexhe (2009) recorded avalanches in cortex of (awake) cats, paying careful attention to the conditions of data collection: avalanche statistics of negative peaks LFP (linked to neuronal firing), positive peaks (unrelated to neuron firing) and surrogate data (obtained by random shuffling experimental data) were analyzed; avalanche criteria were those of the Beggs-Plenz studies. Essentially, time and amplitude distributions of NLFP showed power-law distributions, preferably at high detection thresholds. But, positive NLFP and surrogate data (randomly shuffled peak times - essentially equivalent to a threshold stochastic process - can also show power-law distributed amplitude distributions. The conclusion of this study, then, was that apparent power-law scaling cannot be considered as proof of self-organized criticality.

At this point, a comment seems in order: the publication of Gireesh and Plenz (2008) seems to suggest that cortical layers 2/3 are the preferential site of avalanche occurrence. The data analyzed in the Touboul and Destexhe study were obtained in earlier work of Destexhe et al. (1999) in parietal cortex of awake cats; there is no indication that a possible role of cortical layer was considered. Whether layer specific patterns of neuronal arborization (Callaway, 2002) could be a source of the discrepancy can at this point not be determined.

The discrepancy between presence of $1 / \mathrm{f}$ scaling in ensemble neural activity (EEG and LFP) and, yet, 1/f scaling not being an intrinsic property of the individual neuron itself, that Bedard and El Boustani et al. claimed to have identified, motivated El Boustani et al. (2009) to adopt yet another experimental approach: to this end, they measured the scaling properties of the power-spectrum of the intracellularly recorded membrane potential of individual neurons. The experiments were conducted in cat primary visual cortex in vivo, the animals being anesthetized and paralyzed. Full-field visual stimuli of varying characteristics were presented to the dominant eye to drive the cortical region under study to states with different firing characteristics. The remarkable result was that the frequency scaling of individual cells was largely determined by the visual stimulus statistics. There was no consistent relation between individual neurons' scaling exponent and the visual stimuli, neither was there any correlation between membrane potential and the spiking scaling exponents. Various control tests and a computer model corroborate the authors' conclusion that statistical correlations in a neuron's input (i.e. its presynaptic activity) can modify the power-law exponent of its spiking activity. Hence, it appears that modulation in a neuron's power-law exponent may reflect changes in the correlation state of the network activity. According to these findings, intrinsic cellular properties do not seem to play a major role for its scaling which reflects in the authors's view primarily the network context.

Regarding self-organization, El Boustani and Destexhe (2009) follow the lead taken in Destexhe's Doctoral Thesis of 1992 and observations of Korn and Faure (2003), and present new evidence in support of chaotic dynamics in EEG: sensitivity to initial conditions is of course prominent; it is also associated with broadband powerspectra and a fractal attractor dimension. The authors confront at length the puzzle that coherence and low dimensionality at the macroscopic level of EEG is associated with stochastic neuronal dynamics at the microscopic level. Is this comparable to conditions obtaining in thermodynamics?

Whence criticality? In peripheral neurons, it seems to be favored by closeness to input from peripheral receptors (see Point process analysis in peripheral nerves and neurons). At the mesoscopic level, Plenz and Chialvo's (2009) analysis of avalanches in primate cortex seem to assure legitimate criticality at the mesoscopic level; yet, the work of Bedard et al. (2006a,b) and El Boustani et al. (2009) raises the possibility that scaling properties of neuron activity may not be of intrinsic neuronal origin, but a consequence of network activity. 
Section "Fractals and criticality of brain states" will resume asking: if and where in the nervous system, and under what conditions, does fractality and criticality in the brain originate? What is the nature of their relation? And, how to conceptualize critical behavior?

\section{THE MACROSCOPIC LEVEL OF NEURAL ORGANIZATION Fractals in brain networks}

Fractality at the macroscopic brain level should be viewed in the context of, and in reference to, the two major conceptual and observational frameworks that have come to guide neuroscience research: the network structure of cortical connectivity, and the brain's state of criticality resulting from the complexity of nonlinear dynamic interactions among its constituents. Advances in network theory (Albert and Barabasi, 2002; Dorogovtsev and Mendes, 2001; Park and Newman, 2004) influenced the application of computational and graph-theoretical methods for characterizing structural brain connectivity in accord with statistical and topological criteria (Hilgetag et al., 2002). Examining the columnar organization of neocortical cortex in detail, Roerig and Chen (2002) found that the number of connections to a central neuron has the shape of a long-tailed histogram, fitting a power-law. On the basis of this "biopower-law connection probability function", Stoop and Wagner (2007) tested a range of network types for spread of synchronization among cortical columns: the superiority of the power-law connection was evident. In general, interaction among neurons and neuron ensembles by synchronization is constrained by network topology (Arenas et al., 2008), hence the relevance of network architecture for Neurodynamics. The potential role of neural synchrony for perceptual organization and conscious experience is a subject of a recent review by Uhlhaas et al. (2009). There is considerable evidence that anatomical and functional connections between different cortical areas possess an intricate organization in the form of "small world networks" (Watts and Strogatz, 1998), forming clusters of nearby cortical areas with short links, which in turn have long-range connections to other clusters (Hilgetag and Kaiser, 2004; Sporns and Zwi, 2004; Sporns et al., 2004; Stam, 2004; Stam and Reijneveld, 2007). Neuroanatomical data sets permit identifying a repertoire of characteristic structural building blocks (motifs) (Sporns and Koetter, 2004).

A hierarchical cluster architecture is thought to provide the structural basis for stability and diversity of functional patterns in cortical networks (Kaiser et al., 2007; Kaiser, 2007). Moreover, hierarchical modular topologies assure sustained activation in neural networks, intermediate between rapid fading and generalized activity spread. This can be considered a prerequisite for the occurrence of criticality (Kaiser and Hilgetag, 2010). Hierarchical graphs can switch between different dynamic activity patterns, depending on the level of ongoing (spontaneous) background activity (MüllerLinow et al., 2008; Hütt and Lesne, 2009). In terms of hierarchy theory, these investigations do not specifically address the implications of nested hierarchies which, however, are suggested by the finding of inter- and intra-cluster network hubs (Sporns et al., 2007). Moreover, $\mathrm{fMRI}$ data obtained from subjects in resting state identify strong functional connections between regions for which no direct structural connections are known (Honey et al., 2009). This finding may be an indicator of nested clustering (see Significance of brain criticality and Summary and final thoughts).
In the absence of deliberate external stimulation, neuronal cortical dynamics displays complex spatial and temporal patterns of activity. In simulations of networks that mimic the large-scale inter-areal connection patterns of cortex, activity takes place spontaneously at multiple time scales, punctuated by episodes of inter-regional phase locking of oscillations (Honey et al., 2007). Significantly, the connections link neural populations of multiple levels of scale, from whole brain regions to local cell columns: this suggests that cortical connections may be arranged in fractal, possibly self-similar patterns. Statistical measures of a computational model of a fractal connection pattern did in fact resemble those of a real neuroanatomical data set (Sporns 2006). The computational models also show that varying fractal patterns induce strongly correlated changes in several structural and functional measures of network properties, as evidence of their interdependence.

In general, scale-free complex networks display self-similarity under length-scale transformations (Song et al., 2005) but not necessarily with regard to degree distribution (Kim et al., 2007), but models of scale-free networks need not necessarily be fractal. How, then, can the fractality of many naturally occurring networks come into being? Song et al. (2006) account for the simultaneous emergence of fractality, modularity and small-world effect, as well as the scale-free property of real world networks by a multiplicative growth process: the network growth dynamics is conceived as the inverse of a renormalization procedure, whereby the network hubs accrete connections by linking with less connected nodes, which leads to a robust fractal topology.

Within the small-world network clusters, functional magnetic imaging (fMRI) identifies a scale-free connection pattern inasmuch as the number of links per network node (the node degree) satisfies a power-law relationship (Eguiluz et al., 2005). Likewise, van den Heuvel et al. (2008) find in an imaging study of the resting brain, that inter-voxel connections follow power-law scaling as evidence for scale-free network topology, possibly associated with a smallworld organization. This form of organization is associated with conserved wiring length and conducive to synchronization of activity across the network (Zhou et al., 2007; see also Changizi, 2003; see Neuronal morphology).

Although citing merely a small fraction of the numerous publications concerned with relations between network topology and dynamics, this section underscores two points of relevance for the objective of this review: first, the presence of, and effect on network dynamics of hierarchic network organization (itself being of several types); and, second, effects of network fractality on network dynamics; but the functional implications of the latter, notably for criticality, require further investigation, as does the possibility of self-similar modularity in brain networks. In the case of metabolic networks, the latter is shown to affect path connections for diffusion and resistance of flows (Gallos et al., 2007).

\section{Fractals and criticality of brain states}

Critical activity patterns in brain were cited earlier as the second notable feature in current thinking about global brain function. Brain criticality has a distinguished history. As early as 1950, Turing (1950) postulated that the brain as a dynamical system would operate near a critical state as the prerequisite for instantaneous reaction to novelty. Sudden transitions between stable states of 
motor behavior are well known since the pioneering observations of Haken et al., 1985; Kelso, 1995). The transitions were interpreted as manifestations of metastability in the self-organizing nonlinear dynamic system of the brain, along the theoretical lines formulated in Synergetics (Haken, 1983). Criticality in brain and behavior was first mentioned by Kelso (1984) in a brief note. Using a superconducting quantum interference device (SQUID) sensor array, Kelso et al. (1992) reported a few years later their observations of spontaneous transitions in neuromagnetic field patterns which occur at a critical value of a behavioral parameter: coherent states of both brain and behavior were captured by the spatiotemporal pattern of phase relations among participating components. This was considered evidence for the brain being a pattern forming system that can switch flexibly from one coherent state to another. Chialvo credits also Varela et al. (2001) with the vision of brain large-scale dynamical properties. This is also discussed by Le Van Quyen (2003). Locating cortical regions associated with such phase transitions of motor behavior, Meyer-Lindenberg et al. (2002) showed that TMS can induce switches between two clearly defined and distinct motor behavior patterns. Additional new evidence of aspects of critical brain behavior accrued in rapid sequence in the following years in several forms: as the scale-free connection pattern of cortical networks (Chialvo, 2004; Eguiluz et al., 2005; Chialvo et al., 2008); as result of the avalanche analysis of Beggs and Plenz $(2003,2004)$ at the mesoscopic level; as coordination dynamics of large-scale neural circuits subserving rhythmic sensorimotor behavior (Jantzen et al., 2008); and finally from two fMRI studies at the macroscopic level, which will be reviewed near the end of this Section.

At this point, it seems appropriate to clarify the distinction between the notion of a generic critical cortical state underlying avalanche behavior on the one hand (see The mesoscopic level), and the view of criticality in Critical Theory of Statistical Physics, on the other: both versions of 'criticality' occur in the current Neuroscience discourse. The former is generally viewed as persistent cortical state subject to dynamic transitions associated with the formation and dissolution of attractors (see for instance: Tognoli and Kelso, 2009), related to the notions of Coordination Dynamics. In contrast, critical dynamics in the framework of Critical Theory of Statistical Physics stipulates that the brain is poised to undergo sudden second order phase transitions to new macroscopic configurations with distinctly novel properties. This dynamics is considered universal in its independence of details at the microscopic level (Stanley, 1987; Sornette, 2000). Early-stage activity in the developing retinal network can be cited as an illustrative example (Hennig et al., 2009) of this concept of critical dynamics: rhythmic bursts of action potential in retina ganglion cells, propagating as wave-like events across the retina surface, arise at a very specific network state which meets the criteria of the classical percolation model of Statistical Physics (Essam, 1980). A phase transition consists then in transforming states of purely local into global functional connectedness, the latter displaying long-range correlations and conspicuous fractal properties (Stauffer and Aharony, 1991/1994). Criticality in the framework of Statistical Physics is generally associated with a particular set of ontological implications. Their discussion in Sections "Significance of brain criticality" and "Summary and final thoughts" will leave us with the intriguing (and as yet unresolved) question to what extent, if any, they can be shown to be shared with, or differ from those that would be implicit in the generic brain state view of criticality.

Measuring the fractal dimension of EEG records, Babloyantz (1986) related different values with differences in sleep states. With subjects acting as their own controls, inhalation anesthesia causes a noticeable increase in EEG dimensionality (Mayer-Kress and Layne, 1987). Multichannel MEG records, obtained with a SQUID show scaling with varying degrees of scale similarity, decreasing with the distance between recording channel locations (Novikov et al., 1997). Studying dynamical synchronization in the brain, Gong et al. (2002) find scale invariant fluctuations of dynamical synchronization in human EEG. Linkenkaer-Hansen et al. (2001) report long-range temporal correlations and scaling with $10-20 \mathrm{~Hz}$ brain oscillations. Pursuing this observation in more detail, LinkenkaerHansen (2003) and Linkenkaer-Hansen et al. (2004a) suggest that the long-term spatial-temporal structure of the complex ongoing EEG activity may reflect a memory of the system's dynamics extending beyond just a few seconds, possibly by a continuous modification of functional brain networks in the sense of SOC. In these tests, somatosensory stimuli attenuate temporal correlations and power-law scaling behavior, suggesting that stimuli degrade the network memory of its past. The relationship to SOC was also the subject of the work of Freeman et al. (2003) in measurements of temporal and spatial power-spectral densities that identify EEG phenomena as fractal. Moreover, Freeman (2005) proposed a fieldtheoretic approach to account for scale-free neocortical dynamics. The Power Spectral Density of background Electrocorticogram was found to follow a power-law with exponents between 2 and 4 , reflecting variations in the level of background activity which is thought to be homeostatically regulated by the refractory periods of excitatory neurons (Freeman and Zhai, 2009). In five frequency ranges (extending from 0.5 to $48 \mathrm{~Hz}$ ), detrended fluctuation analysis of EEG show global synchronization time series with scale-free features (Stam and de Bruin, 2004); the scaling exponent differs for conditions of eye open and eye closed. Stam (2005) also reviewed the nonlinear dynamical analysis of EEG and EMG at great length. Positive and negative feedback affect the scaling exponent of EEG differentially; this was determined in a detrended fluctuation analysis (Buiatti et al., 2007). Performance in Stimulus detection of weak stimuli is best accounted for by modulation of the power-law component in the power-spectrum of MEG record: Shimono et al. (2007) attribute this phenomenon to the brain operating in a state of self-organized criticality which modulates the power-spectral exponent to optimize responsiveness to external stimuli.

Transients in EEG records can be detected as differences in fractal dimension of EEG (Arle and Simon, 1990), as can be neuropathological conditions (Paramanathan and Uthayakumar, 2008), and differences in age and gender (Nikulin and Brismar, 2005). Nonlinear spectral analysis enabled Kulish et al. (2006) to determine in EEG a set of generalized fractal dimensions and fractal spectra which reveal differences in subjects when replying to questions with either YES or NO. In a study of human development from infancy to 16 years of age, Thatcher et al. (2009) measured phase shift duration and phase locking intervals of the EEG for computing instantaneous phase differences between pairs of electrodes; the log-log spectral plots showed 1/f distributions. The 
data revealed increased phase stability in local systems, paralleled by lengthened periods of unstable phase relation between distant connections. These results were taken to reflect progression towards self-organized criticality, accompanying the growth spurts from infancy to adolescence. When Listening to music Bhattacharya and Petsche (2001) find homogeneous scaling in the gamma band EEG over distributed brain areas, whereas the homogeneity is reduce at rest, or when reading text or during spatial imagination. As is well known, music has been under scrutiny for fractal properties for quite some time, see for instance: Voss and Clarke (1975), Hsu and Hsu (1991), Boon and Decroly (1995); see also below: Bianco et al. (2007).

The important observations of Lakatos et al. (2005) and their theoretical implications for optimizing the processing of rhythmic stimulus input are of relevance in this context. The laminar analysis of oscillatory field potentials and neuron cluster activity in auditory cortex is the basis for identifying a hierarchical structure in the EEG, with the amplitude at each oscillatory frequency being modulated by the phase of a lower frequency oscillation, whereby higher frequency oscillations reflect excitability variations in neuron clusters. Entrainment of neuronal oscillations is then identified as a mechanism for auditory attention selection, associated with the rhythmic shifting of excitability in local neuronal ensembles (Lakatos et al., 2008).

Two aspects of EEG activity have remained essentially "blind spots" as victims of artificial filtering of brain activity records: Logothetis (2002) and Freeman and Zhai (2009) pointed out that arrhythmic brain activity constitutes a major part of EEG and LFP, about which little is known. For arrhythmic brain activity, this gap is now being remedied by the work of He et al. (2010), demonstrating a power-law relationship in the temporal power spectrum. Moreover, this study reveals extensive nesting of frequencies, with the phase of lower frequencies modulating the amplitude of higher frequencies, in a progression across the frequency spectrum. The power-law exponent varies with brain region and activity. The other neglected dimension of EEG also harbors its surprises: Monto et al. (2008) report that very slow EEG fluctuations in the frequency range of $0.01-0.1 \mathrm{~Hz}$ affect the trial-to-trial behavioral performance and oscillation dynamics, reflecting the excitability dynamics of cortical networks. Cross-frequency synchrony observed by Palva et al. (2005) in Human MEG extends to these very low frequency ranges, as does the scale-free characteristics of cycle length. The phase of ongoing oscillatory EEG activity affects visual perception (Busch et al., 2009), not unlike the enhancement of psychophysical performance by pre-stimulus oscillatory (Linkenkaer-Hansen et al., 2004). Taken together, these observations and those of Lakatos et al. (l.c) show that psychophysical performance or states are sensitively dependent on features of cortical oscillations across a much wider range of frequency and amplitudes than commonly considered. What is of immense interest is that the dynamics of cortical oscillations appears to entail a nested hierarchy of frequency and amplitude interdependencies. Could this relation be a reflection of a cascading functionality, comparable to the principles of linking actions across many scales, discussed in Section "Linking across many scales of space and time"? It would afford the capacity for swiftly shifting between different scales of stimuli on demand and with economy of processing.
Long-range temporal correlations in spontaneous discharge patterns of hippocampal-amygdala complex neurons show a power-law relation in epileptic patients (Bhattacharya et al., 2005): activity of individual neurons was in this study recorded by means of micro-wire electrodes that had been implanted for localization of epileptic foci, and records were taken in inter-ictal periods, with the subjects being awake. Neuronal activity in substantia nigra exhibits fractal activity in anaesthetized rats, but was strikingly absent in the dopaminergic nigrostriatal neurons with relatively constant discharge rate (Rodriguez et al., 2003). The authors consider the possibility that pathological rhythmic discharges and tremor onset may be associated with loss of the fractal pattern of nigrostriatal neurons. During paradoxical sleep and in the attentive state, neurons in the mesencephalic reticular formation of unanesthetized cats exhibit firing patterns with 1/f spectral profile (Yamamoto et al., 1986). Kodama et al. (1989) extended this observation to discharge properties of neurons in Hippocamus and ventrobasal thalamic neurons, and suggest that $1 / \mathrm{f}$ structured patterns in discharge trains are indicative of spatial and temporal summation of convergence. Variations in $1 / \mathrm{f}$ spectra in cortical and subcortical brain structures of monkeys are apparently related to differences in emotional states (Andersen et al., 2006).

In a very detailed and thorough study, Bianco et al. (2007) identify the EEG time series as a (non-ergodic) renewal non-Poisson process, reflecting strong deviation from exponential decay. This startling claim is based on two premises: one, the comparison with the statistics of an entirely different physical process, namely the fluorescence intermittency in blinking quantum dots (Bianco et al., 2005); and, second, on the conjecture of the brain operating at or near a self-organized critical state. The implication is that neuron synchronization can be viewed as a kind of phase transition involving the close cooperation among many constituents of a neuron set, each individual neuron in essence losing its identity. Furthermore, the absence of exponential truncation would violate the ergodic condition (Bel and Barkai, 2005). The authors then proceed to show that compositional music belongs to the same category of processes. They finally claim that the effect of music on the human brain is in fact based on the essential identity of their respective fractal dynamics, ensuing a kind of complexity matching of the interacting brain-music systems. This aspect will be further pursued in Section "Complexity matching effect" Equally consequential are the inferences drawn by Allegrini et al. (2008) from their EEG data. The thrust of their analysis is on measuring the time distribution of recorded events occurring simultaneously at two or more electrodes (in their terminology: coincidences); they find that the time interval between two consecutive coincidences has a waiting time distribution corresponding to perfect $1 / \mathrm{f}$ noise. The theoretical analysis of this finding leads these authors to infer that the coincidences are driven by a renewal process.

The electroencephalographic findings in support of 1/f scaling are supplemented by observations with brain imaging: In 1997, Zarahn et al. (1997) reported BOLD time series data obtained from normal subjects at rest that exhibited a fractal power-spectrum and self-similar signal contributions, with disproportionate contribution of power in the spectrum for low frequencies. The temporal variability of brain activity in time series of fMRI data in combination with a voxel-wise analysis of scaling exponents enabled 
Thurner et al. (2003) to distinguish different physiological states of the brain. In non-active brain regions, the voxel-profile activity is described by a random walk model; in contrast, stimulus activated brain activity is characterized as correlated fractional Brownian noise. The same group of investigators (Shimizu et al., 2004) examined fMRI time series with a multifractal method to extract local singularity (fractal) exponents: the range of Hoelder exponents in voxels with brain activation is close to 1 , whereas exponents in white matter and voxels in the absence of brain activation are close to 0.5.

Without further discussing at this point the far reaching implications of the non-ergodicity claim (Tsallis et al., 1995; Tsallis, 2009), I merely alert to two publications which interpret human EEG signals in terms of a Tsallis Entropy measure (Capurro et al., 1998, 1999).

The common theme of studies surveyed in the following is wavelet-based representations of functional magnetic imaging (fMRI) time series. Among others, Wornell (1993) explicated in detail the role of wavelet-based representations for the power-law family of processes. The remarkable feature of wavelet analysis is that it can be viewed as matching self-similar processes since the wavelet coefficients exactly reproduce, from scale to scale, the selfreplicating statistical structure of such processes (Abry, 2003).

Publishing with various associates since 1994, Bullmore gathered extensive experience with fractal analysis of human brain activity which led eventually to the suggesting that wavelet-based fMRI time series estimates (Bullmore et al., 2001) can be viewed as realizations of Fractional Brownian Motion, i.e., a class of fractals described by Mandelbrot and van Ness (1986), characterized by zero-mean, and non-stationary and non-differentiable time functions (see Power-law scaling in neuronal structures and processes). Extolling further the virtues of wavelet techniques for the purposes on hand, Bullmore et al. (2004) and Maxim et al. (2005) give a meticulous account of their use of the "discrete wavelet transform" approach to fMRI time series evaluation. In normal subjects at rest, the time series is most parsimoniously described as Fractional Gaussian Noise, signifying a persistent long-memory fractal processes of which the Hurst Exponent is a defining parameter. Interestingly, the value of this parameter in Alzheimer subjects differs from the norm (Maxim et al., 2005). Several results from the same laboratory contribute additional facets to the notion of the active brain displaying fractal properties: Achard et al. $(2006,2008)$ applied discrete wavelet transform analysis to fMRI time series to estimate the frequency dependence of functional connectivity between some ninety cortical and subcortical brain regions; the functional networks is dominated by a neocortical core of highly connected hubs with an exponentially truncated power-law degree distribution. Dynamical analysis of brain at wavelet scales from 2 to $37 \mathrm{~Hz}$ show the emergence of long-range connections with execution of motor tasks (Bassett et al., 2006). Under certain conditions (e.g., age, cognitive performance, certain pharmacologic interventions) brain dynamics requires a more comprehensive description than is captured by the monofractal analysis applied in the studies cited thus far (Suckling et al., 2008). In such cases, a more comprehensive description must make allowance for scaling behavior that is governed by several local scaling exponents. Multifractal analysis can then be characterized by the histogram of the Holder exponents. Following expenditure of cognitive effort, the brain's fractal oscillations require several minutes for returning to baseline activity, this time depending on the task's cognitive load; this is taken to signify the relevance of fractal scaling for adaptive task processes, in addition to the role it plays for the "resting" brain (Barnes et al., 2009). The substantial evidence for modular organization of brain networks is reviewed by Bullmore and Sporns (2009), and was subsequently further refined by Meunier et al. (2009), applying a method for rapid, high-resolution modular decomposition of brain functional networks (Blondel et al., 2008). Differences between low frequency BOLD signal spectral power in task and rest periods also support the notion of fMRI reflecting meaningful brain states (Duff et al., 2008), as do the emotional task-dependent fractal fluctuations in fMRI of the cerebellar vermis (Andersen et al., 2006). Brain imaging in Neuropathology has revealed significant differences between patients suffering from unawareness of ownership of one arm (Asomatognosia) and those with additional confabulations (Somatoparaphrenia): the latter patients display lesions in the medial and orbitofrontal regions, in addition to the multiple large lesions including temporo-parietal sectors which are common to both groups of patients (Feinberg et al., 2010). For the distinction of stimuli related to the self (i.e. self-referential stimuli) from those not so related, Northoff et al. (2006) identify processes mediated by cortical midline structures.

Two virtually simultaneously published recent studies, using different experimental strategies, deliver seemingly firm evidence for brain criticality. Kitzbichler et al. (2009) based their approach on the widely accepted view that many behavioral and cognitive states are related to coherent or phase-locked oscillations in transient neuronal assemblies (for a recent summary: Womelsdorf et al., 2007). The measures for determining phase synchronization between component processes were in their study the phase lock intervals (estimating the length of time a pair of bandpass filtered oscillations remain in phase synchronization), and the lability of global synchronization (informally analogous to the previously discussed avalanches). Applying these measures to functional MRI and MEG data recorded from normal volunteers at resting state demonstrated power-law scaling of both pair wise and global synchronization. They then evaluated the performance of two models, both typically being used in nonlinear dynamics: the Ising and the Kuramoto (1984) model. Observed and model generated data were identical, provided the model system was in a critical state. Hence, the authors conclude that the brain must be in a critical state. Moreover, the critical brain dynamics obtained at frequency intervals ranging from $0.05-0.11$ to $62.5-125 \mathrm{~Hz}$, confirming criticality of the human brain network organization across its functional bandwidth. They consider therefore "Broadband Criticality" as a characteristic property of the resting brain network functional organization.

Although also using the Ising model as reference point for determining brain criticality, Fraiman et al. (2009) followed an entirely different approach: the issue at stake in their study was to determine whether and to what extent the dynamics of the paradigmatic two-dimensional Ising model at criticality displays features that correspond to patterns encountered in the imaging of (resting) brain networks. However, unlike most prior studies of brain dynamics cited in this Section, no prior assumptions on structural connectivity of brain regions were made. Instead, network 
connectivity was extracted from voxel correlations: thus, networks were here defined in terms of correlations among the activity at each location (voxel in the case of the brain, and lattice site in the Ising model). Prior investigations showed that the so called "resting state" (absence of overt external stimulation) is subject to a Default Network Dynamics, reflecting balanced positive and negative correlations between activity in component brain regions (Fox and Raichle, 2007; Baliki et al., 2008); this is not the case under certain abnormal conditions (Baliki et al., 2008). The result was that the dynamics of the Ising model at criticality, as captured by the correlation networks, exhibits average statistical properties which are identical to those observed in the brain networks at resting condition. Among several other network characteristics that match critical Ising dynamics with brain dynamics was also the equality of the fraction of sites with positive and negative correlations, corroborating that the dynamics of the normally functioning brain at rest being near a critical point. In any case, the unequivocal answer to the question the investigators set out to answer was that networks derived from correlations of fMRI signals in human brains are indistinguishable from networks extracted from Ising models at critical temperature.

In an important next step, Expert et al. (2010) investigated the large-scale dynamical properties of resting brain by examining more closely the character of the spatio-temporal correlations: considering three successive steps in spatial coarse graining, twopoint correlation functions exhibit self-similarity; self-similarity in time was revealed by $1 / \mathrm{f}$ frequency behavior of the power-spectrum. The condition of long-range correlations in space and time presupposes a dynamical system at criticality; the strong correlations across large distances are indicators of highly integrated cortical states, with nearby clusters functioning in synchrony.

Apart from this principal conclusion of this study, the authors also alert to a significant property of the brain networks which, as noted before, are extracted from the site-to-site temporal voxel correlations: obviously, equally oriented spins in the Ising model coalesce in large domains near the critical temperature where also nontrivial collective states emerge in the Ising model's otherwise regular lattice. Similarly, large regions of brain activate concurrently with deactivation of other regions. How does the brain selforganize to negotiate the dynamic balance between the extreme possibilities of total quiescence and explosive massive excitation? The authors refer to a discussion of this stability problem which was already noted by Abeles (1991). It motivates their question: is it necessary to confine brain activation to structural connections linking brain regions, as is customary in most current research? (e.g.: Hagmann et al., 2008). Take the Ising model as example: there, a change of temperature can lead to the emergence of functional collectives, in the absence of preexisting structural connections. This leads Fraiman et al. to ask: might the brain, likewise, have this capacity, as basis of a kind of adaptive coordination analogous to that envisioned by Kelso and Tognoli (2007) and Tognoli and Kelso (2009)?

Apparently, SOC, metastability and critical phase transitions constitute a nexus of intimately interrelated dynamic processes of which fractals and self-similarity are pivotal aspects (Bak and Paczuski, 1995). I already alerted in earlier Sections to the distinction between critical cortical states as generic brain feature and phase transitions in the framework of Critical Theory of Statistical Physics. When Kitzbichler et al. (l.c.) and Fraiman et al. (l.c.) consider the Ising dynamics as reference points for identifying brain criticality, they invoke the framework of equilibrium phase transitions in Statistical Physics; on the other hand, and in line with a related comments in Section "The mesoscopic level", "critical cortical state" may be more closely related to Kelso's evocative notion of 'exploring the space of possibilities' among competing (conflicting) dynamic regimes (Kelso, 2010; see also: Kelso and Engstrøm, 2006) than to "critical phase transitions" of Statistical Physics. I raise this issue to alert to the need for making the distinction between two kinds of dynamical regimes under discussion: one, conceptually related to the theory of 'Coordination Dynamics' (see Kelso and Tognoli, l.c), the other aligned with Critical Theory of Statistical Physics. The latter implies a distinct ontological commitment, commonly associated with the Renormalization Group Framework. The next section will develop a view of Brain Criticality in terms of the Critical Theory. It then remains an important question to what extent, if any, the ontological implications of Critical Theory would also apply to critical brain states as persistent and generic brain features, or what their intrinsic nature is.

\section{Significance of brain criticality}

In statistical Physics, systems operating at the critical point of transition between ordered and random behavior are metastable with respect to a set of control parameters, and are capable of rapid qualitative change in response to fluctuations of external input. For systems far from equilibrium most of the analytical and numerical methods of the 'classical' (equilibrium) theory appear to remain valid (Sornette, 2000). Moreover, dissipative (open) Hamiltonian System, such as the brain, have the capacity to form "strange" attractors whose boundaries and bases have fractal properties (Aguirre et al., 2009; see Processes that generate power-law distributions). At or near the point of phase transition, the systems exhibit complex patterns of fluctuations on all scales of space and time, this being one of the indicators of an impending phase transition; another indicator is the slowing down of relaxation processes, associated with forming long-range correlations for efficient functional coupling among system components: both events are anticipatory signals of impending critical transitions (Scheffer et al., 2009). Fractal clusters formed by phase transitions can be characterized in terms of correlation length (Antoniou et al., 2000) which is associated with fractal scaling of clusters of correlated elements on all scales; as a result, any intrinsic scale before phase transition is de facto 'forgotten' (Stinchcombe, 1989). As a corollary, the system presents at the critical transition qualitatively new properties, requiring new macroscopic descriptors. The important feature of the organization following the phase transition is to form new objects with distinct properties. In physics, this is manifest as, for instance, the phase transition from ferro- to para-magnetism, or from water to ice (Stanley, 1999). Typically, one deals with a large collection of 'microscopic' constituents which, at phase transitions, arrange to a macrostate which displays qualitatively novel features and properties. The macrostate's new properties have no referent at the microscopic level, and require new descriptors: by way of illustration, think of hardness or liquidity in the ice-water example as descriptors of new physical properties, originating de novo 
upon phase transition. The properties described in the foregoing are universal in the sense that the apply irrespective of the system's constituents at the microscopic level.

One of the amazing features of phase transitions is that material systems of diverse physical properties at their microscopic level form on phase transition but a small number of Universality Classes which share identical macroscopic properties (for a discussion in relation to brain function: see Werner, 2009c). Based on a stochastic theory of neural activity, Buice and Cowan (2007) developed field-theoretic methods for non-equilibrium statistical processes; their model exhibits a dynamical phase transition of the universality class of directed percolation (see Fractals and criticality of brain states).

Critical Theory (Stanley, 1987; Yeomans, 1992; Marro and Dickman, 1999) considers reality as a hierarchy of levels, each having its own scale, its own description and a theory that accounts for that description. The scale on each level emerges from the scale on the next finer level by ignoring some of the lower level details which become invisible at the higher level scale (Sokal and Bricmont, 2004; Laughlin, 2005). The result is a drastic reduction of dimensionality. Coarse graining (specifically Renormalization Group Transformation) (Fisher, 1998) unveils self-similarity at the point of phase transition. The intimate relations between scaling, renormalization group, and long-range correlations are addressed by Perez-Mercader (2004) and Penrose (1986), the latter pointing out that the definition of fractal dimension depends primarily on the distribution of widely separated sites, telling little on sites that are close together.

What is the significance of criticality? Excitable systems at criticality exhibit an optimal dynamical range for information processing (Kinouchi and Copelli, 2006; Beggs, 2008; see Shew et al., 2009, in The mesoscopic level). Furthermore, a model that reproduces the typical features of systems at a critical point learns and remembers complex logical rules: learning occurs by plastic adaptation of synaptic strengths, and exhibits universal features in being independent of the specific task assigned to the system (de Arcangelis and Herrmann, 2010). Finally, phase transition in critical systems are a universal mechanism for rapid switching between different cooperative neuron collectives. These three attributes of criticality are the reason for its rapidly moving into center stage of current brain theory. A remarkable consequence of phase transitions is the generation of qualitative novelty in the form of macrostates with new properties: Section "Summary and final thoughts" will discuss this aspect in relation to brain theory.

\section{PSYCHOLOGICAL AND BEHAVIORAL PROCESSES}

The following overview of psychological functions with power-law scaling is predicated on the notion that mental states may be viewed as macrostates emerging from EEG dynamics (Allefeld et al., 2009), and neurophysiological processes generally. Classical Psychophysics of Helmholtz, Fechner and Weber sought to establish dependencies of perceptual experience on properties of physical stimuli impinging on sensory organs. In 1975, Stevens reported the summary of the extensive work that led him to propose that this dependency is in many sensory modalities a power-function. In neurophysiological experiments, Werner and Mountcastle $(1963,1964)$ identified the powerfunction scaling of responses in primary afferent cutaneous nerve fibers to mechanical indentation of peripheral receptors. Neurons of primary visual cortex (V1) exhibit a higher coding efficiency and information transmission rate for input signals with natural longterm (1/f) correlations (Yu et al., 2005). Copelli et al. (2002) and Kinouchi and Copelli (2006) claim that Stevens (1957) law for intensity of subjective sensory experiences can be attributed to dynamics in a network of excitable elements constituting the peripheral receptors, set at the edge of a phase transition, i.e.: of being in a state of criticality. For a discussion of this view, see Chialvo (2006).

Unlike dismissing the fluctuations in the performance of many psychophysical task as “noise", Gilden $(1997,2001)$ attributes them to a memory process associated with active choice and discrimination. This memory process is suggested to express itself as $1 / \mathrm{f}^{\prime}$ 'noise' in the three major measurement paradigms in Psychophysics: speeded judgment, accuracy of discrimination and production. The 1 /f fluctuations are attributed to an intrinsic dynamics, associated with the formation of representations, comparable to the kind of memory that arises in dynamical systems as they flow forward in time, along principles outlined by Beran (1994). According to this interpretations of the psychophysical observations, cognition would generate its dynamical signature as a consequence of its own activity: this would entail a fundamental revision of what is signal and what is noise in psychophysical data. Gilden points out that the conventional experimental design and data analysis using ANOVA does in fact bury "one of the most important signatures of what happens when the mind is working".

Timing fluctuations in tasks requiring sensorimotor coordination display cycle-to-cycle fluctuations which, analyzed as time series, show fractal scaling of power-spectra (Chen et al., 1997). Ding et al. (2001) suggest that the reason for this lies in the multiple time scale activities of distributed neural areas that contribute to the task performance. If asked to produce random series of numbers from a given set, series with short and long-range correlations are produced which in most cases exhibit a power-law spectrum (Morariu et al., 2001). Van Orden et al. (2003) interpret serial correlations in human cognition as evidence of self-organization. In their view, self-organization coordinates the activities of the organism across a hierarchy of time scales, producing correlated variation across time: variations in response times would then appear as a natural fractal in which larger scale deviations nest within themselves smaller (self-similar) scale deviations. Accordingly, 1/f noise is in this view not sufficient evidence for self-organized criticality, but rather its necessary consequence. Similarly, Kello et al. (2007) assembled reaction time and response data which lead them to considering the $1 / \mathrm{f}$ scaling of their data as expression of a coordinative, metastable basis of cognitive functions. This view is in effect an extension of Van Orden's et al. (2003), shifting the genesis of 1/f scaling from self-organization to metastability: the 1/f pervasiveness in the brain would be the signature of metastability associated with cognitive functions. However, these claims are challenged by Wagenmakers et al. (2005) and contrasted with the alternative that long-term serial dependence in data can be explained in a number of ways, for instance by mixtures of a small number of short-range processes) (Wagenmakers et al., 2004).

Applied to problem solving and insight, reasoning was viewed by Stephen and Dixon (2009) as the self-organization of novel structures: taking a particular problem solving task as example, the 
authors suggest that the problem solution can be viewed as a phase transition in a self-organizing system whose dynamics would be reflected in power-law behavior. Implications for social psychology are reviewed by Correll (2008): cognitive effort to avoid bias in judgments reduces the scaling exponents of response times relative to less challenging tasks. Grigolini et al. (2009) interpret Correll's data to suggest that increasing the difficulty of cognitive tasks would accelerate the transition from observed $1 /$ f noise to white noise in decision making time series.

The temporal structure of many human-initiated activities can display a striking regularity. Barabasi (2005) showed that a decision-based queuing process can account for the dynamics of some human patterns of activity: when individuals execute tasks based on some perceived priority, the timing of the tasks will indicate the signatures of fractal dynamics: heavy-tailed distributions with initial fast bursts.

If patterns of expression in spoken language reflect in some way the organization of brain processes, then Zipf's law is of course the notable landmark that presages more recent fascinating reports of fractal patterns and scale-invariant word transition probabilities in spoken and written texts (Alvarez-Lacalle et al., 2006; Altmann et al., 2009; Costa et al., 2009), and their extension to music (Zanette, 2008). On the basis of EMG data, it appears that some common features of patterning in language, music and syntax (Patel, 2003) can be attributed to neural activity in Broca's area and its right hemisphere homolog (Maess et al., 2001).

\section{SYMBOL PROCESSING AND FRACTALS}

The classical book "Language of Thought" (Fodor, 1975) epitomizes the framework of computation-representation of the Computational Theory of Mind. However, with adopting a dynamical perspective, it became appropriate to view "representation" in terms of regions of state space, and "computational rules" as attractors (Elman, 1995); the dynamics is supplied by recurrent neural networks (RNN). Systems of this kind learn to recognize and generate languages after being trained on suitable examples. Surprisingly, it turned out that the induction of this ability occurs when small network parameter adjustments bring about a phase transition in the neural network's state space. Once in a certain state, machine states for correct recognitions scale with an exponent of 1.4 (Pollack, 1991). Considering the RNN as a dynamical system, it appears that its trajectories can locate regions in phase space which support fractal dynamics: putting it in a graphical way, the phase space would seem "peppered" with regions for fractal dynamics (i.e. attractors), which can be reached by the trajectories of the complex system's dynamics. Numerous additional sources point to a close, though often not readily transparent relation between the dynamics of RNN and IFS: the principle is consistent with the observations of Pollack (1991) inasmuch as fractal sets provide a method for organizing recursive computation in a bounded state space (Tabor, 2000). Furthermore, context-free grammar computation by connectionist networks using fractal sets can generate spatial representations of symbolic sequences (Jeffrey, 1990; Tino, 1999) via IFS (Barnsley and Demko, 1985; Barnsley et al., 1989). A class of associative reinforcement learning algorithms was constructed by Bressloff and Stark (1992) as an extension of non-associative schemes in stochastic automata theory; within the IFS framework, it suggested a possibly fractal nature of the learning process. Tsuda and Kuroda (2004) recently elaborated this idea and developed a mathematical model of Cantor Coding for the formation of episodic memory in the hippocampus.

The intent is here merely to draw attention to a large segment of literature, of which the foregoing citations are but a small sample that implicates interrelations between state space dynamics of RNN and IFS in the processing of symbolic information. For clarification of this relationship, Kolen $(1994,2006)$ proposed that the RNN's state dynamics itself is an IFS, as a paradigmatic case of the synergism of fractal and complex system dynamics. Levy and Pollack (2002) obtained supportive evidence in that every point in the hidden layer of the RNN is either itself part of the fractal attractor of the IFS, or has an orbit that "ends on" the attractor in a finite number of steps. Two tantalizing questions arise: one, wherein does the "computational" power of a Fractal System lie? How does the self-similar structures of fractals unpack layers of "information" for guiding actions across many scales still eludes our comprehension. And, second, what exactly is the nature of that apparent synergism between complex system and fractal dynamics? (see Linking across many scales of space and time).

\section{MOTOR BEHAVIOR AND ALLOMETRIC CONTROL PROCESSES}

During quiet standing, the human body sways in a seemingly erratic fashion. Collins and De Luca (1994) determined that the pattern of this postural sway is exhibits intrinsic correlations which can be modeled as a system of bounded correlated random walks. This result suggested to the authors that the postural control system incorporates both open and closed loop control mechanisms. The statistics of temporal patterns in spontaneous motor activity of laboratory rodents can be replicated by the stochastic mechanism of Davidsen and Schuster (2002, see Processes that generate power-law distributions) which generates powerlaw distributions and $11 / \mathrm{f}$ power-spectrum over several decades (Anteneodo and Chialvo, 2009). The presence of long-time correlations in the stride-interval time of normal humans suggested to Hausdorff et al. (1995) that the activity of walking may be a self-similar fractal. Acknowledging the complexity of locomotor activity, the authors referred to its prerequisite of coordinating inputs from motor cortex, basal ganglia, and cerebellum, as well as feedback from vestibular, visual, and proprioceptive sources. In the Hausdorff et al. study, the gait cycle (synonymous with stride interval) was defined as the time between consecutive heel strikes of the same foot.

West and Griffin (1999) and Griffin et al. (2000) took a different and novel approach to the analysis of gait patterns: using the time between consecutive maximal positive extensions of the same knee for measuring the stride interval, their data analysis was based on determining the long-time correlation properties of the stride-interval time series. The Relative Dispersion (given as the ratio of standard deviation to the mean) for different levels of data aggregation captures the inter-relatedness of the data across multiple time scales. The method is described in detail by Bassingthwaighte et al. (1994) and is designed to answer whether the correlations are self-similar upon scaling (i.e.: identical between groups of neighbors at different time scales). The result of the data analysis was that the fluctuations of the gait cycle were self-similar 
with a fractal dimension of 1.25 . In addition, stride-interval time series itself was in this study a random fractal, consistent with the data of Hausdorff et al.

The importance of the West-Griffin results lies in showing that the correlation in their data was an inverse power-law of a form similar to the allometric scaling laws found in many areas of Biology: typically, allometry establishes a relation between two properties of an organism. Historically, the idea is based on Huxley's (1932) definition of allometric growth, describing that the different growth rates of two parts of an organism are proportional to one another. In the West-Griffin studies, the allometric principle is reflected in the constancy of the Relative Dispersion over the length of the stride-interval time series which, in the present case, has the non-integer fractal dimension of 1.25. Their data raise the issue of systematic control of variability, which is generic of complex physiological processes. Unlike the familiar homeostatic control that regulates system variables by negative feedback, an allometric control system is conceived as regulating variability of a process involving multiple interactions among sensors and effectors with intricate feedback arrangements, each with its own characteristic set of frequencies and time scales. Their functions are reflected by the allometric relation which captures the process's long-term memory with power-law correlations, and by the power-law distributions of the system variable (West, 1999, 2009).

The significance of this principle is documented by West (1999, 2006) for the numerous physiological processes which are identified as fractal, on the basis of their time series behavior. Notable examples of fractal Physiology are heart rate, bronchial air ways and body temperature variability, and integrated neural control networks. In these situations, the regulatory mechanisms constitute coupled cascades of feedback loops in systems far from equilibrium. Therapeutic interventions, commonly based on the homeostatic principle which assumes the significant system variable to be normally distributed fails to take the regulatory complexity into account and may be counterproductive. Instead, Allometric (fractional) Control based on Fractional Calculus (Podlubny, 1999) provides the appropriate approach. Applications of Fractional Calculus to modeling the interdependence and organization of complex system, such as for instance the vestibulo-oculomotor system, are illustrated by Magin (2006).

Changing walking speed, using metronomically controlled walking, or aging and pathological conditions introduce stress conditions to the neural control system which requires expanding the theoretical framework. Based on the notion of a stochastic model of human gait dynamics (Ashkenazy et al., 2002), West and Scafetta (2003) tested the model of a neural pattern generator on the data set obtained by Hausdorff et al. (1995) which they showed to exhibit slightly multifractal fluctuations. Metronome timing breaks the long-time correlations of the natural pace and generates a large fractal variability of the gait regime. The two essential features of the model required for capturing the phenomenology of the data set were that the dynamics of the system unfolds on an attractor in phase space, and that the natural frequency of the attractor is replaced by a random walk over a restricted set of frequencies which leads to the multifractal output for the dynamical model (Scafetta et al., 2009).

\section{PROCESSES THAT GENERATE POWER-LAW DISTRIBUTIONS}

Mitzenmacher (2003) reviewed some aspects of the rich and long history of generative models for power law and log normal distributions, and their relations. Antedating the modern theory of stochastic processes, Yule (1925) proposed a model of speciation to explain the highly skewed distributions of abundances of biological genera. Thirty years later, Simon (1955) derived several related stochastic processes from relatively general probability assumptions that lead to Yule-type distributions. Their characteristic properties distinguish them from the negative binomial and Fisher's logarithmic series. Leaving open the possibility of still other generative mechanisms for power-law distributions, Simon suggests that the frequency of occurrence of this empirical distribution should not come as surprise. The preferential attachment scheme for network growth (Barabasi and Albert, 1999) has stimulated the recent interest in the Yule-Simon approach in as much as Bornholdt and Ebel (2001) could show that they are closely related. The important step of introducing the notion of aging of network nodes was taken by Dorogovtsev and Mendes (2000): the probability of being linked to a newly added node is taken to be proportional to its current connectivity weighted by a power-law function of its age. This motivated Cattuto et al. (2006) to propose a modified Yule-Simon process that takes the full history of the system into account, applying a hyperbolic memory kernel.

Simon's conclusion that power-law distributions can be derived from relatively general assumption seems to be born out by the number of mathematical models that have been proposed. A shotnoise process, reviewed by Milotti (2002) is an example, as is the Reversible Markov Chain Models (Erland and Greenwood, 2007), and the Clustering Poisson Point Process (Grüneis, 2001), the latter already introduced in the Section "Power-Law Scaling in Neuronal Structures and Processes." The simple stochastic mechanism of Davidsen and Schuster (2002) generates pulse trains with power-law distributions of pulse intervals, and $1 / \mathrm{f}$ power-spectra over several decades at low frequencies with an exponent close to 1. Iterated function systems (IFSs) are a unified approach for generating and classifying a broad class of fractals with self-similarity (Barnsley and Demko, 1985). The Chaos Game is a generalized form of this, designating a method for generating the attractor (fixed point) of any IFS. Other Recurrence Models (Kaulakys and Meskauskas, 1998; Kaulakys et al., 2006) derive from a more specific frame of reference insofar as they consider random walks in complex systems that display self-organization. As alternative, Ruseckas and Kaulakys (2010) generate $1 / \mathrm{f}$ noise with nonlinear stochastic differential equations. Touboul and Destexhe (2009) followed a similar route when developing their case against power-law scaling of neural avalanches (see The mesoscopic level). Physical systems whose observable properties exhibit values which randomly exceed certain critical values are candidates for applying Extreme Value Theory: the aim of the classical form of this theory is to quantify the properties of the extremes (large or small) occurring in random sequences of independent numbers. Extremal dynamics may be applied to generate objects with fractal structure (Miller et al., 1993); as Extremal Optimization, it successively eliminates undesirable components of suboptimal problem solutions (Boettcher and Percus, 2000).

The various approaches discussed in the foregoing can essentially be viewed as ad hoc (Milotti, 2002). In contrast, however, there are two types of conceptual anchors that ground power-law relations 
explicitly in larger foundational contexts. For one of the conceptual roots, I turn to the theory of Random Walks and fractional difference equations. The continuum limit of simple random walks is diffusion and, correspondingly, expressed in the mathematics of differential equations. The simple random walk aggregates the random steps from a large number of identically distributed random variables with finite variance. However, an extensive range of investigations has made it abundantly clear that simple random walks with this statistics do not capture the richness of biological data, and for that matter other fields of investigation as well (for reviews see: Bassingthwaighte et al., 1994; West and Deering, 1995; West 1999). A decisive step beyond simple random walks was the introduction of the concept of continuoustime-random walk (CTRW) by Montroll and Weiss (1965). Some forms of CTRW are fundamentally different from the classical diffusion model by drawing the timing of steps from waiting time distributions, or by taking steps of randomly varying length. This is for instance the case when the waiting time distribution does not possess a characteristic time scale (for instance, has a power-law distribution): in this situation, the mean square displacement and the distribution of transition rates become fractal. Processes corresponding to these and related random walk models are then referred to as fractal random walks, corresponding to anomalous diffusion which occupies an important place for studying physical processes such as transport in disordered media or non-exponential (anomalous) relaxation of, for instance, glassy media. Along these lines, Montroll and West, 1979), Hughes et al. (1982) and others examined a large repertoire of stochastic processes with unusual probability distributions for the displacement per step. For certain parameters, these walks have infinite spatial moments, generate fractal self-similar trajectories, have characteristic functions with non-analytic behavior, and lead to an analog of RNG transformations. In the continuum limit, the fractal random walk leads to the Fractional Langevin Equation of motion describing trajectories, and their ensemble densities, in phase space (West, 2006). Such processes are viewed as fractional kinetics, and mathematically addressed in fractional calculus (Kleinz and Osler, 2000; West and Nonnenmacher, 2001; Sokolov et al., 2002) and by Fractal Operators (West et al., 2003).

In an application to Neuroscience, Lundstrom et al. (2008) showed that neocortical pyramidal neurons' firing rate is a fractional derivative of slowly varying stimulus parameters: neuronal fractional differentiation effectively results in adaptation with many time scales (see Linking across many scales of space and time). Fractional-order dynamics of brainstem vestibulo-oculomotor neurons was demonstrated by Anastasio (1994) who also suggested that simulation of fractional-order differentiators and integrators can be approximated by integer-order high- and low-pass filters, respectively. Thus, fractional dynamics may possibly be applicable to motor control systems, generally. This is also suggested by the stride-interval time series of human gait being a random fractal, indicating the role of long-time correlations in walking (West and Griffin, 1999; see Symbol processing and Fractals). Mandelbrot and van Ness (1968) defined Fractional Brownian Motions as a family of Gaussian random functions, parametrized according to the interdependence of successive increments, with the parameter ranging from zero (Gaussian Fractional Random Walk) to infinite in Fractional Brownian motion: the latter to account for the empirical studies of random phenomena with interdepend- ence of distant samples. The conceptual connections to scaling invariance and to the theory of renormalization are discussed by Quian (2003). Fractional reaction-diffusion in inhomogenous media stabilizes steady state solutions of Turing patterns (Henry and Wearne, 2000).

Shlesinger et al. (1987) introduced the Levy walk as a random walk with non-local memory, coupling space and time in a scaling fashion. For the alpha-stable Levy Walks, the transition probability varies with the size of the step (Montroll and West, 1979). Anomalous diffusion results from a Levy Flight which is a process where the time taken to complete a transition depends on the length of the step (West et al., 1997). West et al. (1994) also identified dynamical generators of Levy Statistics. In an elegant step towards unifying various classes of random walks, Zumofen and Klafter (1993) applied the framework of CTRW's to derive Levy stable processes. The interesting properties of Levy processes include their satisfying a scaling law, self-similarity and possessing memory (Allegrini et al., 2002). Levy (1954) also generalized the Central Limit Theorem to include those phenomena for which the second moment diverges. West and Deering (1995) and West (2006) assembled a large number of data obtained from various biological systems that satisfy Levy walk statistics. In a motor skill acquisition task, Cluff and Balasubramaniam (2009) report that probability distributions for changes of fingertip speed in pole balancing are Levy distributed. In vitro recorded spontaneous electrical activity of neuronal networks exhibits scale - invariant Levy distributions and long-range correlations (Segev et al., 2002). This is thought to enable different size networks to self-organize for adjusting their activities over many time scales. Among animal movement patterns associated with random search behavior, Levy walks outperform fractional Brownian motion (Reynolds, 2009), presumably evolved under section pressure (Bartumeus, 2007). Situating the power-law formalism in the larger framework of Maximum Entropy, Frank (2009) develops the view of the power-law as the outcome of one particular form of aggregation of processes, namely aggregation that preserves information only about the geometric mean. More generally, this important study places the power-law alongside other generative, each placing its distinct constraints on information for describing patterns of nature.

Physical process models to account for fractal heavy-tailed distributions of traffic pattern of (information) packages in local area networks (LANs) are based on renewal reward processes, originally applied to commodity pricing. Applied to network package traffic, the model takes into account the presence of long packet trains ("on periods", with packages arriving at regular intervals) and long inter-train pauses ("off periods"). The superposition of many such packet trains displays on large time scales the self-similar behavior LANs if the "on-off" distribution has infinite variance (Willinger et al., 1995; Willinger, 2000).

The second conceptual framework was already introduced in Section 2.3.3: power-law distributions are among the novelties that arise in the vicinity of or at the critical point of a continuous phase transition, including criticality. This should not come as surprise since scaling reflects long-time correlations in the underlying process, analogous to the comparable re-ordering process at critical phase transitions (Wilson, 1979): both cases address a class of phenomena where events at many scales make contributions of equal 
importance. Significantly, the comprehensive review on Fractal structures in nonlinear dynamics by Aguirre et al. (2009) begins with the sentence "Fractal structures appear naturally in nonlinear dynamics, in such a way that the two concepts are deeply related". Their review draws particular attention to numerous instances in nature where attractor basin boundaries in dissipative (open) systems display fractal behavior. Gisiger's (2001) comment is a propos: "at one point, Bak (1996) considered SOC as universal, with scaling as consequence; it appears, however, that the balance of evidence shifted the question: why is there scale invariance in Nature? to the question: is Nature critical?"

For constructing theories that deal with problems that have multiple scales, the renormalization group (RNG) offers a general method. In Physics, the most frequently studied situation is "percolation transition" for which Newman (2005) offers a detailed account of the origin of power-law scaling: the cumulative distribution of cluster sizes forms at the critical point a power-law distribution. Percolation transition is a special case under the closely interconnected family of RNG and coarse graining that entails power-law distributions as a source of natural fractals (see Fractals and Criticality of Brain States). Coarse graining allows one to determine whether the phenomenon under investigation has universality, apart from scaling: Universality implies that macroscopic properties of a system are independent of the system's particular microscopic configuration. The particular values determined for a given instantiation of the system are then not significant, apart from showing that the system scales: the theoretical foundations are extensively discussed by Essam (1980) and by Stauffer and Aharony (1991/1994).

For Neuroscience, Kozma et al. (2005) illustrated the potential relevance of percolation for phase transitions in models of neural populations with mixed local and global interactions, and (Werner, 2009b,c) proposed Renormalization Group Transformation as a general principle to account for functional relations between levels of neural organization. Since fractals will in both situations naturally arise, it is pertinent to ask what their role could be. West et al. (2008) and Allegrini et al. (2006a,b) attribute to them a complexity matching function which will be the subject of review and comments in the Section "Complexity matching effect".

\section{FRACTALS IN ACTION}

Having reached the end of the largely phenomenological surveys of fractal scaling and associated manifestations of fractality at the conventionally distinguished levels of organization and function of nervous systems, it appears inescapable to recall the title of Barnsley's (1993) book "Fractals everywhere". The apparent prevalence in the nervous system is matched by the numerous manifestation in physiological systems generally (West and Deering, 1995). Is the ubiquity a sign of triviality, or the result of a generic and fundamental principle of Nature? If that is the case in Biology at least, then Nature seems to adhere to it with remarkable conservatism as, for instance, the monographs of Dewey (1999) for Molecular Biophysics and of Seuront (2010) in Ecology attest.

As documented by Aguirre et al. (2009), and is referenced in several sections of this review: the intimate relation between fractals and nonlinear dynamics in dissipative systems is apparent and well substantiated. Hence the seemingly disproportionate attention paid to phase transitions and criticality in Sections "Peripheral nervous system" and "The mesoscopic level". However, Section "Processes that generate power-law distributions" lists a large number of alternatives for generating fractals, some of which obviously qualifying as "natural", as, for instance Levy flights. Thus, the burden of proof of attributing observed fractals to nonlinear dynamics lies on identifying the fractal boundaries or the critical phase transition that gave rise to them. This subset of natural fractal must then be viewed as manifestation of, and a consequence of, the particular dynamic regime from which they originated.

\section{THE COMPLEXITY MATCHING EFFECT (CME)}

The issue under consideration is the communication among complex systems generating fractal signatures. The starting point is the evidence presented in Section 1.4 that the EEG time series can be identified as a (non-ergodic) non-Poisson renewal (NPR) process, reflecting strong deviation from exponential decay. A brief account of CME will suffice at this point since a comprehensive overview of the underlying principle of CME is available in West et al. (2008). CME is concerned with the conditions under which one complex network responds to a perturbation by a second complex network: Consider a NPR network with a power-law index $<2$ as measure of its complexity, and apply a random signal as perturbation: this is in essence comparable to the condition of aperiodic Statistic Resonance (Gammaitoni et al., 1998). Allegrini et al. (2006a,b) then generalized the conditions by applying as perturbation another complex network which also satisfies the NPR condition with power-I law index $<2$. Under these conditions, it can be shown that the effect of the perturbation is maximal if the power-law indices of the interacting systems are equal. The claim is that CME, as illustrated in the foregoing, applies to a large class of NPRs such as, for instance, return times for random walks, either in regular lattices or in complex networks.

\section{LINKING ACTIONS ACROSS MANY SCALES?}

Even if Nature's conservative adherence to fractals is a valid argument in support of their functional significance, we are still in the dark as to what that function may be. Emphasizing the feature of self-similarity, we can turn to types of functions which could benefit from stacking extended ranges of space and/or time scales into one compact format; moreover, there is no privileged time scale in power-law dynamics. Sensory adaptation as a change over time in the responsiveness of the sensory system to a constant stimulus is a situation of this kind (Wark et al., 2008). Adaptation with powerlaw dependence and multiple time scales has been demonstrated in nervous systems under many different conditions. Examples come from such diverse sources as electrosensory afferent nerve fibers in weakly electric fish (Xu et al., 1996), spider mechanoreceptor neurons (French and Torkkeli, 2008). The auditory sensory memory which is thought to encode stimuli on multiple time scales (Ulanovsky et al., 2004). Fairhall et al. (2001a,b) direct attention to the speed with which the dynamics of a neural code is optimized, even when the statistical properties of the stimuli themselves evolve dynamically over a wide range of timescales, from tens of milliseconds to minutes. The source of $1 / \mathrm{f}$ fluctuations in human sensorimotor coordination tasks is presumably attributable to the multiple time scale activities of neural centers (Ding et al., 2001). In visual psychophysics, adaptation to contrast follows power-law 
dynamics (Rose and Lowe, 1982) as does the tilt aftereffect (Greenlee and Mangussen, 1987). For fractional-order dynamics in adaptation, see: Lundstrom et al. (2008) and Anastasio (1994) in Section "Processes that generate power-law distributions".

This fragmentary compilation of diverse observations is intended to illustrate the propensity of various neural structures to respond swiftly to a range of temporal and/or spatial parameters: the image of a set of strings resonating to specific frequencies comes to mind. Could self-similar fractal structures, having stored a repertoire of responses, each specific for a particular range of temporal and/ or spatial stimulus parameters, fulfill this function? On formal grounds, Thorson and Biederman-Thorson (1974) attributed sensory adaptation to distributed relaxation processes, based on non-uniformities of local "efficacy" in the transduction process at peripheral receptors. Might this "local non-uniformity" be the expression of a self-similar functionality of receptors?

Generically, models of adaptation integrate the response of a system and feed the integrated signal back to curtail that response. The type of adaptation is determined by the properties of the integrator. Applying this principle, Drew and Abbott (2006) examined a form of power-law integration with the result that the suppressive effect of repeated stimuli on successive responses are accumulated with power-law decline. In distinction from other forms of integration (e.g.: exponential), power-law integration has the notable feature of scale-invariance and, in their simulations, replicated the published data of Xu et al. (1996), cited in the previous paragraph. On the same principle, these investigators also implemented power-law adaption within a standard spiking neuron model, except for approximating the time dependence of the power-law adaptation integral for computational efficiency by a series exponentials. This principle has been successfully applied by Hausdorff and Peng (1996) and is the basis of Anderson's (2001) assertion of the power-law being an emergent function: it amounts to linking the non-interacting exponential processes to a cascade which reproduces the power-law forgetting in power-law integration. The adaptation obtained of the integrate-and-fire model with adaptation current obtained by a cascade of exponential processes matched perfectly that obtained by injecting adaptation currents to the model neurons.

The implications of this successful approach are considerable: the Dell and Abbott results suggest that power-law adaptation can be instantiated by a cascade of a large number of processes with ordinary exponential dynamics, covering a wide range of time constants. The cascaded model design lets the temporal stimulus dynamics set the appropriate adaptation dynamics, in virtue of the numerous exponential processes with different time scales. They present a telling argument in support of the biological significance of this mechanism of adaptation: natural stimuli vary unpredictably over a wide of time scales; instead keeping the recovery time after excitation constant, power-law adaptation allows the temporal statistics of the stimuli themselves to determine the dynamics of adaptation. The work of Toib et al. (1998) and Gilboa et al. (2005) referred to in Section "Ion channels" are additional examples of "multiscale computing" involving fractals.

Are we prepared to envision a general principle of self-organizing control structures for multiscale behavior, extracting the statistics of an unpredictable environment by way of power-law integration? The papers cited in the next paragraph speak to this question.
Fusi et al. (2005) applied the principle of power-law forgetting of adaptation to a cascade model for regulating the plasticity of synapses as the basis of stored memories. Note that memory strength is here represented as synaptic plasticity, not as synaptic strength, as is commonly the case. Each synapse model has two levels of synaptic strength, weak and strong. Associated with each strength is a cascade of states (in one of their models, five). The cascades introduce a range of probabilities for transition between weak and strong levels of synaptic plasticity. A complicated heuristics is built into the model design that enables combinations of states of plasticity, for instance: states with low probability are paired up with labile states, and conversely, etc. The important point is that a high level of memory storage with long retention times significantly outperforms other model designs. In a model of learning visuomotor associations that are reversed unpredictably from time to time, synaptic modification occurring on multiple time scales along the same principles can be the basis for flexible behavior; the model predictions were validated with experimental data (Fusi et al., 2007). For the possibility of cascading structures for EEG phase and amplitude, see Section "Fractals and criticality of brain states", and references to Lakatos et al. (l.c.), He et al. (l.c.), and Mono et al. (l.c.).

It appears then, that the principle of shifting between different scales on demand manifests itself in many different forms, and on different levels of neural organization. Under natural conditions, are the required exponential functions for power-law integration models of self-similar structures?

\section{SUMMARY AND FINAL THOUGHTS}

The assembly of largely phenomenological data was presented to support the claim that fractal processes and properties occur at many and diverse levels of neural organizations and performance, and are functionally relevant. Several issues that must not glossed over lightly needed discussion in several places: e.g. fractality as such is not an obligatory indicator of SOC; whether non-conservative systems may be limited to a state of quasi-criticality instead of being candidates for full criticality (see Peripheral nervous system); and what the origin of natural fractals may be, including their standing in a Maximum Entropy framework (Frank, 2009; see "Processes that generate power-law distributions"). In Sections "Fractals and criticality of brain states" and "Significance of brain criticality", the tantalizing question was addressed that discourse on criticality may in different contexts refer to different dynamical regimes. However, whichever stance is taken, the close relation of fractals with critical dynamics is beyond dispute. Despite the evidence secured by Plenz and Chialvo (1.c.) in specially targeted experiments, critical dynamics is still by some investigators called in question at the mesoscopic level, but the evidence for its importance and essential role at the macroscopic domain is uncontroversial and solid.

Among the virtues of brain criticality in the sense discussed in Section "Significance of brain criticality", there is one that has attracted attention for the longest time: it is the ability for rapid changes of state. Note that the following considerations pertain to the Phase transition in the framework of Critical Theory, as a universal mechanism for rapid switching between different cooperative neuron collectives. Less attention received the fact, well established in Physics, that the system presents at the critical 
transition qualitatively new properties, requiring new macroscopic descriptors (for more details: Werner, 2009c). The important feature of the organization following the phase transition is to form new objects with distinct properties: a new Ontology. In Physics, transitions between ferro- and para-magnetism are the prototypical example. We need to ask: What, if any, is the manifestation of ontological novelty in this form of brain phase transition? Customarily, one tends to think in terms of integration and differentiation, brought about by the change in correlation patterns of the system components, with nearby clusters functioning in synchrony. However, this way of looking at the nature of the state change fails to meet the requirement for qualitative (ontological) novelty, as the analogy with Physics would require. In the paradigmatic cases in Physics, new aggregations are being formed on phase transition which display novel properties at a macroscopic level of description. What can we assume to happen in brain on phase transition? The obvious answer is: partitioning into neural assemblies with fractal properties. This is of course merely analogous to the fractal patterns formed at phase transition in physical systems. But here is an essential difference between the systems studied in Physics and the brain: Take the ferro-paramagnetic phase transition again as example, it consist merely in a change of spin orientation of the elementary components. But in the case of brain, the elementary components are reactive neurons which have the potential of entering into aggregations with functional interactions. Model studies of de Arcangelis and Herrmann (2010) with self-organizing neuronal networks show that avalanches formed at phase transition can learn complex rules on the basis of a collective process. Under appropriate conditions, the learning dynamics is universal inasmuch as even complex rules can be acquired. Recalling Section "Symbol processing and fractals", the relation of the state dynamics of these neural networks to IFSs and fractal attractors would constitute a notable case of the synergism of fractal and complex system dynamics.

Returning now to the "other" of critical brain dynamics which views brain criticality as generic and persistent state: do any of these or other implications of ontological import pertain to state transitions in this dynamic regime?

Re-visiting the work of Beggs and Plenz (see The mesoscopic level) and putting it bluntly: phase transition (viewed in the framework of Critical Theory) would endow avalanches with novel capacities that are not analytically predictable from the original state of the system. Generalizing from this, it is evident that pursuing these and related directions in targeted studies of the aftermath of brain phase transitions is imperative for seeking to gain a full appreciation of the functional novelties it may create. Whether such studies will show a way of bridging the ultimate barrier of the epistemic cut (Pattee, 2001) that separates the domain of integrable Physics from the domain of symbolic structures remains to be seen: but this issue is, I submit, at the core of the apparent object-subject (brain-mind) duality.

The limited sample of recent publication on network models cited in Section "Fractals in brain networks" is but a fraction of the large number of combinations and permutations of network design parameters that are conceivable: on the one hand, ranging from the of small-world to scale-free works with various degree distributions, on other hand each type being hierarchical, modular with and without hubs, and fractal. Which one from among this collection is closest to the natural brain networks? Suggestions abound, but tantalizing questions remain still open to further inquiry. An architecture that does justice to self-similarity under renormalization appears attractive as it might unite the dynamics of network topology in one common mechanism, as I suggest in that Section.

Commenting on the wealth of existing data on anatomical and functional cortical networks organization may seem like "carrying coals to Newcastle", notably in view of the comprehensive review prepared by Bullmore and Sporns (2009). Nevertheless, a few general considerations may be of some relevance. It is a commonplace observation that Nature loves Hierarchies: in the influential article "Architecture of Complexity", Simon (1962) gave plausible reasons for this apparent love affair. A few years later, in a discussion of complex systems, Simon (1973) observed that the term "hierarchy" has taken a somewhat generalized meaning, divorced from its original denotation in human organizations of a vertical authority structure. He there contended that in application to complex systems, "Hierarchy" has come to denote a set of Chinese Boxes of a particular kind, usually consisting of their recursion. But then, these Chinese Box hierarchies come in variants of one form or another of ordering, e.g. in the partial ordering of trees. Finally, in the 3rd edition of "The Sciences of the Artifical", Simon (1996) settled on applying the term "Hierarchy" in a much broader sense, referring to all complex systems that are analyzable into successive sets of subsystems: this entails partitioning in conjunction with the relations that hold among the subsets (l.c., p. 197). For this historical and a variety of other reasons, there are now diverse (and sometimes ambiguous) senses in current use by investigators reporting their finding in anatomical, $\mathrm{fMRI}$ and model studies when speaking of hierarchical order. A precise definition for fitting a hierarchical structure to the topology of a graph is proposed by Clauset et al. (2006).

The network and graph-theoretical community has developed its own criteria: modularity has been introduced a measure of a networks decomposability, for instance into community structures (Newman and Girvan, 2004), possibly at different hierarchical levels, allowing one to "zoom in and out" for finding communities on different levels; this was discussed in detail by Meunier et al. (l.c.). Mucha et al. (2009) seek to address this same objective in a new framework that encompasses coupling of individual networks via links connecting nodes of one with nodes of another network at multiple scales, thus allowing for some nesting which escapes the Newman-Girvan method, but is accessible by the approach of Sales-Pardo et al. (2007). Keeping these issues in mind is, I suggest, of importance in the definitive evaluation of many of the reports of fMRI data on cortical functional organization: while describing modularity, they may not be able to capture nesting among modules, due to methodological limitations. Yet, it is the nesting of the kind that Simon refers to as "Russian doll" (see also the Russian Matryoshka dolls of Agnati, l.c.), where the pressing question of self-similarity arises: It has some plausibility in view of Sporns's (2006) suggestive evidence for cortical connection patterns of fractal and self-similar nature. In different contexts, self-similar community structures were noted by Guimera et al. (2003) in a network of human interactions, and nesting of theta- and beta gamma oscillations was noted by Gireesh and Plenz (2008) in neuronal 
avalanches of developing cortical layers $2 / 3$. Efforts continue to seek consensus on criteria for modules, communities, and subgraphs in networks, and the methodology for their delineation (see for instance: Shalizi et al., 2006; Lambiotte et al., 2009, 2010).

The foregoing discussion was merely intended to draw attention to some of the problems involved in attempts to characterize dynamic hierarchies of a system that consists of multiple levels of organization, having dynamics within and between the entities described at each of the different levels (Lenaerts et al., 2005). Hierarchical nesting would presumably qualify brain dynamics as a dynamic hierarchy. This is consonant with the recent findings of Honey et al. (2009) of indirect functional connectivity, not accounted for by structural connections, and the variability of resting-state functional connectivity across scanning sessions and model runs. As regards Criticality, it would then be imperative to examine the properties of phase transitions in dynamically hierarchic systems. In one recent study of a kinetic Ising model, it turned out that the universality class is consistent with the twodimensional equilibrium Ising model (Rikvold et al., 1999).

Sadly, despite the resourceful and imaginative studies undertaken by many investigators during the past decade, we are still some distance away from understanding the structural and functional basis of cortical dynamics; let alone the cortical-subcortical dynamics that, according to the Global Workspace Hypothesis (Baars, 1988) is now generally considered decisive for cognitive functions.

\section{REFERENCES}

Abbott, L. F., and Rohrkemper, R. (2007). A simple growth model constructs critical avalanche networks. Prog. Brain Res. 165, 13-19.

Abeles, M. (1991). Corticonics: Neural Circuits of the Cerebral Cortex. Cambridge: Cambridge University Press.

Abry, P. (2003). Scaling and Wavelets: an introductory Walk. Lect. Notes Phys. 621, 34-60.

Achard, S., Bassett, D. S., MeyerLindenberg, A., and Bullmore, E. (2008). Fractal connectivity of longmemory networks. Phys. Rev. E 77, 036104.

Achard, S., Salvador, R., Whicher, B., Suckling, J., and Bullmore, E. (2006). A resilient, low-frequency, small-world human brain functional network with highly connected association cortical hubs. J. Neurosci. 26, 63-72.

Agnati, L. F., Baluska, F., Barlow, P. W., and Guidolin, D. (2009). Mosaic, self-similarity logic, and biological attraction. Commun. Integr. Biol. 2, 552-563.

Agnati, L. F., Santarossa, L., Genedani, S., Canela, E. I., Leo, G., Franco, R., Woofs, A., Lluis, C., Ferre, S., Fuxe, K. (2004) "On the nested hierarchical organization of CNS: basic characteristics of neuronal molecular organization," in Cortical Dynamics LNCS 3146 (ed. P. Erdi), 24-54, Springer, Berlin.
Aguirre, J., Viana, R. L., and Sanjuan, M. A. F. (2009). Fractal structures in nonlinear dynamics. Rev. Mod. Phys. 81, 333-386.

Albert, R., and Barabasi, A. (2002). Statistical mechanics of complex networks. Rev. Mod. Phys. 74, 47-97.

Allefeld, C., Atmanspacher, H., and Wackermann, J. (2009). Mental states as macrostates emerging from brain electrical dynamics. Chaos 19, 015102.

Allegrini, P., Bellazzini, J., Bramanti, G., Ignaccolo, M., Grigolini, P., and Yang, J. (2002). Scaling breakdown: a signature of aging. Phys. Rev. E 66, 015101.

Allegrini, P., Bologna, M., Grigolini, P., and West, B. J. (2006a). Response of complex systems to complex perturbations: the complexity matching effect. arXiv:cond-mat/0612303v1.

Allegrini, P., Bologna, M., Grigolini, P., and Lukovic, M. (2006b). Response of complex systems to complex perturbations: complexity matching. arXiv:cond-mat/0008341vl.

Allegrini, P., Menicucci, D., Bedini, R., Fronzoni, L., Gemignani, A., Grigolini, P., West, B. J., and Paradisi, P. (2008). Spontaneous brain activity as a source of perfect 1/f noise. PNAS 98, 694-699.

Altmann, E. G., Pierrehumbert, J. B., and Motter, A. E. (2009). Beyond word frequency: bursts, lulls, and scaling in the temporal distributions of words. arXiv:0901.2349v1.

Notwithstanding this disappointing state of affairs, one more speculations may perhaps be permitted: recall the amazing discovery from Section "Linking across many scales of space and time": there, power-law forgetting of adaptation and synaptic modification on multiple time scales were discussed as principles for flexible behavior. Recall also the evidence from Section "Ion channels" for multiscale computing as basis cooperative fractal channel kinetics. The essential principle in these cases is the capacity of having available the responsiveness, on demand, to a large range of unpredictable external contingencies of a certain category, varying in one parameter: e.g., the capacity for selecting the level of adaptation appropriate to a wide range of temporal patterns and stimulus intensity. In the cases referred to, it amounted to scale shifting as required, involving fractality and self-similarity of control structures. If Sporns's evidence for cortical fractality and self-similarity holds up, I can now re-visit the claim I made in the Introduction: "is there a natural capacity for unpacking interactions between different levels of a fractal object or process, responsive to circumstances and conditions, which eludes us entirely? If it existed, fractals would surely be a most extraordinary design principle for operational economy in complex systems."

To which I now add: if this natural capacity embodies principles of power-law integration and cascading, similar to those identified for adaptation and fractal kinetics, we may come to comprehend at least some aspects of the extraordinary functionality of the cortex.

Alvarez-Lacalle, E., Dorow, B., Eckmann, J.-P., and Moses, E. (2006). Hierarchical structures induce long-range dynamical correlations in written texts. Proc Natl. Acad. Sci USA, 103, 7956-7961.

Anastasio, T. J. (1994). The fractional-order dynamics of brainstem vestiblo-oculomotor neurons. Biol. Cybern. 72, 69-79.

Andersen, C. M. (2000). From molecules to mind: how vertically convergent fractal time fluctuations unify cognition and emotion. Conscious. Emot. 1, 193-226.

Andersen, C. M., Lowen, S. B., and Renshaw, P.F. (2006). Emotional taskdependent low-frequency fluctuations and methylphenidate: wavelet scaling analysis of $1 / \mathrm{f}$-type fluctuations in fMRI of the cerebellar vermis. J. Neurosci. Methods 151, 52-61.

Anderson, R. B. (2001). The power-law as an emergent property. Mem. Cognit. 29, 1061-1068.

Anteneodo, C., and Chialvo, D. R. (2009). Unraveling the fluctuations of animal motor activity. Chaos 19, 1

Antoniou, N. G., Contoyiannis, Y. F., and Diakonos, F. K. (2000). Fractal geometry of critical systems. Phys. Rev. E, 62,3 .

Arenas, A., Diaz-Guilera, A., Kurths, J., Moreno, Y., and Zhou, C. (2008). Synchronization in complex networks. Phys. Rep. 469, 93-153.

Arle, J. E., and Simon, R. H. (1990). An application of fractal dimension to the detection of transients in the electroencephalogram. Electroencephalogr. Clin. Neurophysiol. 75, 296-305.

Ashkenazy, Y., Hausdorff, J. M., Ivanov, P. Ch., and Stanley, E. (2002). A stochastic model of human gait dynamics. Physica A 316, 662-670.

Baars, B. J. (1988). A Cognitive Theory of Consciousness. Cambridge: Cambridge University Press.

Babloyantz, A. (1986). "Evidence of chaotic dynamics of brain activity during the sleep cycle," in Dimensions and Entropies in Chaotic Systems quantification of complex behavior, ed. G. Mayer-Kress (Berlin: Springer), 114-122.

Baddeley, R., Abbott, L. F., Booth, M. C.A., Sengpiel, F., Freeman, T., Wakeman, E. A., and Rolls, E. T. (1997). Responses of neurons in primary and inferior temporal visual cortices to natural scenes. Proc. R. Soc. Lond. B, 264, 1775-1783.

Bak, P. (1996). How Nature works: the Science of self-organized criticality. New York: Springer.

Bak, P., and Paczuski, M. (1995). Complexity, contingency, and criticality. Proc. Natl. Acad. Sci. U.S.A. 92, 6689-6696.

Bak, P., Tang, C., and Wiesenfeld, K. (1987). Self-organized criticality: an explanation of $1 / \mathrm{f}$ noise. Phys. Rev. Lett. 59, 381-384.

Baliki, M. N., Geha, P. Y., Apkarian, A. V., and Chialvo, D. R. (2008). Beyond 
feeling: chronic pain hurts the brain, disrupting the default-mode network dynamics. J. Neurosci. 28, 1398-1403.

Barabasi, A-L. (2005), The origin of bursts and heavy tails in human dynamics. Nature 435, 207-211.

Barabási, A. L., and Albert, R. (1999). Emergence of scaling in random networks. Science 286, 509-512.

Barnes, A., Bullmore, E. T., and Suckling, J. (2009). Endogenous human brain dynamics recover slowly following cognitive effort. PLoS One 4, e6626. doi:10.1371/journal.pone.0006626.

Barnsley, M. F. (1993).Fractals Everywhere. New York: Academic Press.

Barnsley, M. F., and Demko, S. (1985). Iterated function systems and the global construction of fractals. Proc. R. Soc. Lond. A, 399, 243-275.

Barnsley, M. F., Elton, J. H., and Hardin, D. P. (1989). Recurrent iterated function systems. Constr. Approx. 5, 3-31.

Bartumeus, F. (2007). Levy processes in animal movement: an evolutionary hypothesis. Fractals 15, 151-162.

Bassett,D.S., Meyer-Lindenberg,A.,Achard, S., Duke, T., and Bullmore, E. (2006). Adaptive reconfiguration of fractal small-world human brain functional networks. PNAS, 103, 19518-19523.

Bassingthwaighte, J. B., Liebovitch, L. S., and West, B. J. (1994). Fractal Physiology. New York: Oxford University Press.

Bedard, C., Kroeger, H., and Destexhe, A. (2006a). Model of low-pass filter of local field potentials in brain tissue. Phys. Rev. E 73, 051911.

Bedard, C., Kroeger, H., and Destexhe, A. (2006b). Does the $1 / f$ frequency scaling of brain signals reflect selforganized critical states? Phys. Rev. Lett. 97, 118102.

Beggs, J.M. (2007). How to build a critical mind. Nat. Phys. 3, 834-835.

Beggs, J.M. (2008). The criticality hypothesis: how local cortical networks might optimize information processing. Phil. Trans. R. Soc. A 366, 329-343.

Beggs, J.M., and Plenz, D. (2003). Neuronal avalanches in neocortical circuits. $J$. Neurosci. 23, 11167-11177.

Beggs, J. M., and Plenz, D. (2004). Neuronal avalanches are diverse and precise activity patterns that are stable for many hours in cortical slice cultures. J. Neurosci. 24, 5216-5229.

Bel, G., and Barkai, E. (2005). Weak ergodicity breaking in the continuoustime random walk. Phys. Rev. Lett. 94, 240602.

Beran,J. (1994). Statisticsfor Long-Memory Processes. New York: Chapman \& Hall.

Bernard, F., Bossu, J-L., and Gaillard, S. (2001). Identification of living oligodendrocyte developmental stages by fractal analysis of cell morphology. $J$. Neurosci. Res. 65, 439-445.

Bhattacharya, J., Edwards, J., Mamelak, A. N., and Schuman, E. M. (2005). Long-range temporal correlations in the spontaneous spiking of neurons in the hippocampal-amygdala complex of humans. Neuroscience 131, 547-555.

Bhattacharya, J., and Petsche, H. (2001). Universality in the brain while listening to music. Proc. R. Soc. Lond. B, 268, 2423-2433.

Bianco, S., Grigolini, P., and Paradisi, P. (2005). Fluorescence intermittency in blinking quantum dots: renewal or slow modulation? J. Chem. Phys. 123,174704

Bianco, S., Ignaccolo, M., Rider, M. S., Ross, M. J., Winsor, P., and Grigolini, P. (2007). Brain, music, and nonPoisson renewal process. Phys. Rev. E, 75, 061911.

Bieberich, E. (2002). Recurrent fractal neural networks: a strategy for the exchange of local and global information processing in the brain. BioSystems 66, 145-164.

Blondel, V. D., Guillame, J-L., Lambiotte, R., and Lefebvre, E. (2008). Fast unfolding of communities in large networks. J. Stat. Mech. Theory E $1 \mathrm{P} 10008$.

Bloom, J.L. (1969). Quantitative Aspects of Neural Activity in the Cerebral Cortex of the Rabbit. Ph.D. thesis, Amsterdam University, Netherlands.

Boettcher, S., and Percus, A. (2000). Nature's way of optimizing. Artif. Intell. 119, 275-286.

Bonachela, J.A., and Munoz, M.A. (2009). Self-organization without conservation: true or just apparent scale invariance? J. Stat. Mech. 9, 1-37.

Boon, J. P., and Decroly, O. (1995). Dynamical systems theory for music dynamics. Chaos 5, 501-508.

Bornholdt, S., and Ebel, H. (2001). World wide web scaling exponent from Simon's 1955 model. Phys. Rev. E, 64, 0351004.

Breskin, I., Soriano, J., Moses, E., and Tlusty, T. (2006). Percolation in living neural networks. Phys. Rev. Lett. 97, 188102.

Bressloff, P. C., and Stark, J. (1992). Analysis of associative reinforcement learning in neural networks using iterated function systems. IEEE Trans. Man Cybern. 22, 1348-1360.

Buiatti, M., Papo, D., Baudonniere, P.-M., and Vreeswijk, C. V. (2007). Feedback modulates the temporal scale-free dynamics of brain electrical activity in a hypothesis testing task. Neuroscience 146, 1400-1412.

Buice, M. A., and Cowan, J. D. (2007). Field-theoretic approach to fluctua- tion effects in neural networks. Phys. Rev. E 75, 051919

Bullmore, E., Fadili, J., Maxim, V., Sendur L., Whitcher, B., Suckling, J., Brammer, M., and Breakspear, M. (2004) Wavelets and functional magnetic resonance imaging of the human brain. Neuroimage 23, S234-S249.

Bullmore, E., Long, C., Suckling, J., Fadili, J., Calvert, G., Zelaya, F., Carpenter, T. A., and Brammer, M. (2001). Colored noise and computational inference in neurophysiological (fMRI) time series analysis: resampling methods in time and wavelet domains. Hum. Brain Mapp. 12, 61-78.

Bullmore, E., and Sporns, O. (2009). Complex brain networks: graph theoretical analysis of structural and functional systems. Nature Reviews Neurosci. 10, 186-198.

Busch, N. A., Dubois, J., and Van Rullen, R. (2009). The phase of ongoing EEG oscillations predicts visual perception. J. Neurosci. 29, 7869-7876.

Callaway, E. M. (2002). Cell type specificity of local cortical connections. $J$. Neurocytol. 31, 231-237.

Capurro, A., Diambra, L., Lorenzo, D. Macadar, O., Martin, M. T., Mostaccio C., Plastino, A., Perez, J., Rofman, E., Torres, M. E., and Velluti, J. (1999). Human brain dynamics: the analysis of EEG signals with Tsallis information measure. Physica A 265, 235-254.

Capurro, A., Diambra, L., Lorenzo, D., Macadar, O., Martin, M. T., Mostaccio, C., Plastino, A., Rofman, E., Torres, M. E., and Velluti, J. (1998). Tsallis entropy and cortical dynamics: the analysis of EEG signals. Physica A 257, 149-155.

Caserta, F., Eldred, W. D., Fernandez, E., Hausman, R. E., Stanford, L. R. Bulderev, S. V., Schwarzer, S., and Stanley, H. E. (1995). Determination of fractal dimension of physiologically characterized neurons in two and three dimensions. J. Neurosci. Methods $56,133-144$

Caserta, F., Stanley, H. E., Eldred, W. D., Daccord, G., Hausman, R. E., and Nittman, J. (1990). Physical mechanisms underlying neurite outgrowth: a quantitative analysis of neuronal shape. Phys. Rev. Lett. 64, 95-98.

Cateau, H., and Reyes, A. D. (2006) Relation between single neuron and population spiking statistics and effects on network activity. Phys. Rev. Lett. 96, 058101.

Cattuto, C., Loreto, V., and Servedio, V. D. P. (2006). A Yule-Simon process with memory. arXiv:condmat/0608672v1

Cessac, B. (2004). Some fractal aspects of self-organized criticality. arXiv:nlin/0409004v1.
Changizi, M.A. (2001a). Principles underlying mammalian neocortical scaling. Biol. Cybern. 84, 207-215.

Changizi, M. A. (2001b) Universal scaling laws for hierarchical complexity in languages, organisms, behaviors and other combinatorial systems. $J$. Theoret. Biol. 211, 277-295.

Changizi, M. A. (2003). The Brain from 25,000 Feet. Dordrecht: Kluwer.

Chen, D., Wu, S., Guo, A., and Yang, Z. R. (1995). Self-organized criticality in a cellular automaton model of pulse-coupled integrate-and-fire neurons. J. Physics A 28, 5177-5182.

Chen, Y., Ding, M., and Kelso, J. A. S. (1997). Long memory processes (1/ fo type) in human coordination. Phys. Rev. Lett. 79, 4501-4504.

Chialvo, D. R. (2004). Critical brain networks. Physica A 340, 756-765.

Chialvo, D. R. (2006). Are our senses critical? Nat. Phys. 2, 301-302.

Chialvo, D. R., Balenzuela, P., and Fraiman, D. (2008). The brain: what is critica about it? Conf. Proc. Am. Inst. Phys. $1028,28-45$.

Clauset, A., Moore, C., and Newman, M. (2006). "Structural inference of hierarchies in networks," in Proc. 23rd Int. Conf. Mach. Learn. Pittsburgh, PA.

Clauset, A., Shalizi, C. R., and Newman, M. E. J. (2009). Power-law distributions in empirical data. arXiv:0706.1062v2.

Cluff, T., and Balasubramaniam, R. (2009). Motor learning characterized by changing levy distributions. PLoS One 4, e5998. doi:10.1371/journal. pone.0005998.

Collins, J. J., and De Luca, C. J. (1994). Random walking during quiet standing. Phys. Rev. Lett. 73, 764-767.

Copelli, M., Roque, A. C., Oliviera, R. F., and Kinouchi, O. (2002). Physics of psychophysics: Stevens and WeberFechner laws and transfer functions of excitable media. Phys. Rev. E, 65, 060901

Corner, M. A., van Pelt, J., Wolters, P. S., Baker, R. E., and Nuytinck, R. H. (2002). Physiological effects of sustained blockade of excitatory tynsotic transmission on spontaneously active developing neuronal networks - an inquiry into the reciprocal linkage between intrinsic biorhythms and neuroplasticity in early ontogeny. Neurosci. Biobehav. Rev. 26, 127-185.

Correll, J. (2008). 1/f Noise and effort on implicit measures of bias. J. Pers. Soc Psychol. 94, 48-59.

Cosenza, M. G., and Kapral, R. (1992). Coupled maps on fractal lattices. Phys. Rev. A. 46, 1850-1858.

Costa, M. E., Sigman, M., and Bonomo F. (2009). Scale-invariant transition probabilities in free word association 
trajectories. Front. Integr. Neurosci. 3, 19. doi:10.3389/neuro.07.019.2009.

da Silva, L., Papa, A. R. R., and de Souza, A. M. C. (1998). Criticality in a simple model for brain functioning. Phys. Lett. A, 242, 343-348.

Davidsen, L., and Schuster, H. G. (2002). Simple model for $1 / \mathrm{f}$ noise. Phys. Rev. E. 65, 026120 .

de Arcangelis, L., and Herrmann, H. J. (2010). Learning as a phenomenon occurring in a critical state. arXiv:1003.1200v1 [q-biol.NC].

de Arcangelis, L., Perrone-Capano, C., and Herrmann, H.J. (2006). Self-organized criticality for brain plasticity. Phys. Rev. Lett. 96, 028107.

de Carvalho, J. X., and Prado, C. P. C. (2000). Self-organized criticality in the Olami-Feder-Christensen model. Phys. Rev. Lett. 84, 4006-4009.

DelCastillo, J., and Katz, B. (1954). Quantal components of the end plate potential. J. Physiol. (Lond.) 124, 560-573.

Delignieres, D., Ramdani, S., Lemoine, L., Torre, K., Fortes, M., and Ninot, G. (2006). Fractal analyses for 'short' time series: a re-assessment of classical methods. J Math. Psychol. 50, 525-544.

De Los Rios, P., and Zhang, Y.-C. (1999). Universal $1 / \mathrm{f}$ noise from dissipative self-organized criticality models. Phys. Rev. Lett. 82, 472-475.

Destexhe, A., Contreras, D., and Steriade, M. (1999). Spatiotemporal analysis of local field potentials and unit discharges in cat cerebral cortex natural wake and sleep states. J. Neurosci. 19, 4595-4608.

Dewey, T. G. (1999). Fractals in Molecular Biophysics. New York: Oxford University Press.

Ding, M., Chen, Y., and Kelso, J. A. S. (2001). Statistical Analysis of timing errors. Brain Cogn. 48, 98-106.

Dorogovtsev, S. N., and Mendes, J. F. F. (2000). Evolution of networks with aging of sites. Phys. Rev. E 62, 1842-1845.

Dorogovtsev, S. N., and Mendes, J. F. F. (2001). Evolution of networks. Adv. Phys. 51, 1079-1187.

Dorogovtsev, S., and Mendes, J. (2003). Evolution of Networks: From Biological Nets to the Internet and $W W W$. New York: Cambridge University Press.

Drew, P. J., and Abbott, L. F. (2006). Models and properties of power-law adaptation in neural systems. J. Neurophysiol. 96, 826-833.

Duff, E. P., Johnston, L. A., Xiong, J., Fox, P. T., Mareels, I., and Egan, G. F. (2008). The power of spectral density analysis for mapping endogenous BOLD signal fluctuations. Hum. Brain Mapp. $29,778-790$.
Eguiluz, V.M., Chialvo, D. R., Cecchi, G.A, Baliki, M., and Apkarian, A.V. (2005). Scale-free brain functional networks. Phys. Rev. Lett. 94, 018102.

Eke, A., Herman, P., Kocsis, L., and Kozak, L. R. (2002). Fractal characterization of complexity in temporal physiological signals. Physiol. Meas. 23, R1-R38.

El Boustani, S., and Destexhe, A. (2009). Does brain activity tem from highdimensional chaotic dynamics? Evidence from the human electroencephalogram, cat cerebral cortex and artificial neuronal networks. arXiv:0904:4217v1 [nlin.CD].

El Boustani, S. E., Marre, O., Behuret, S., Baudot, P., Yger, P., Bal, T., Destexhe, A., and Fregnac, Y. (2009). Network-state modulation of power-law frequencyscaling in visual cortical neurons. PLoS Comput. Biol. 5, e1000519. doi:10.1371/journal.pcbi.1000519.

El Boustani, S., Pospischil, M., RudolphLilith, M., and Destexhe, A. (2007). Activated cortical states: experiments, analyses and models. J. Physiol. (Paris) 101, 99-109.

Elman, J.L. (1995). "Language as a dynamical system," in Mind as Motion, eds R. F. Port and T. Gelder (Cambridge, MA: MIT Press), 192-225.

Erland, S., and Greenwood, P. E. (2007) Constructing $1 / \omega^{\alpha}$ noise from reversible Markov chains. Phys. Rev. E, 76, 031114.

Essam, J. W. (1980). Percolation theory. Rep. Progr. Phys. 43, 834-912.

Eurich, C. W., Herrmann, J. M., and Ernst, U. A. (2002). Finite size effects of avalanche dynamics. Phys. Rev. E 66:066137.

Evarts, E. V. (1967). “Unit activity in sleep and wakefulness," in Neurosciences, Neurosciences Research Program, eds G. C. Quarton, T. Melnechuk, and F. O. Schmitt (New York: Rockfeller University Press), 545-556.

Expert, P., Lambiotte, R., Chialvo, D. R., Christensen, K., Jensen, H. J., Sharp, D. J., and Turkheimer, F. (2010). Selfsimilar correlation function in brain resting state fMRI. arXiv:1003.3682v1 [q-biol.NC].

Fairhall, A. L., Lewen, G. D., Bialek, W., and van Steveninck, R.R. (2001a). Multiple timescales of adaptation in a neural code. Adv. Neural Inform. Proc. Syst. $13,124-130$.

Fairhall, A. L., Lewen, G. D., Bialek, W., and de Ruyter van Steveninck, R. R. (2001b) Efficiency and ambiguity in an adaptive neural code. Nature 412, 776-777.

Feinberg, T. E., Venneri, A., Simone, A. M., Fan, Y., and Northoff, G. (2010). The neuroanatomy of asomatognosia and somatoparaphrenia. J. Neurol Neurosurg. Psychiatr. 81, 276-281.
Feng, J., and Zhang, P. (2001). Behavior of integrate-and-fire and HodgkinHuxley models with correlated inputs. Phys.Rev. E, 63, 051902.

Fisher, M. E. (1998). Renormalization group theory: its basis and formulation in statistical physics. Rev. Mod. Phys. 70, 653-681.

Fodor, J. (1975). Language of Thought. Cambridge, MA: Harvard University Press.

Fox, M. D., and Raichle, M. E. (2007) Spontaneous fluctuations in brain activity observed with functional magnetic imaging. Nat. Rev. Neurosci. 8, 701-711.

Fraiman, D., Balenzuela, P., Foss, J., and Chialvo, D. R. (2009). Ising-like dynamics in large-scale functional brain networks. Phys. Rev. E, 79, 061922

Frank, S. A. (2009). The common patterns of Nature. J. Evol. Biol. 22, 1563-1585.

Freeman, W. J. (2005). A field-theoretic approach to understanding scale-free neocortical dynamics. Biol. Cybern. 92, 350-359.

Freeman, W. J., Holmes, M. D., Burke, B. C., and Vanhatalo, S. (2003). Spatial spectra of scalp EEG and EMG from awake humans. Clin. Neurophys. 114 1053-1068.

Freeman, W. J., and Zhai, J. (2009) Simulated power spectrum density (PSD) of background electrocorticogram (ECoG). Cogn. Neurodyn. 3, 97-103.

French, A. S., and Torkkeli, P. H. (2008) The power-law of sensory adaptation: simulation by a model of excitability in spider mechanoreceptor neurons. Ann. Biomed. Eng. 36, 153-161.

Fusi, S., Asaad, W. F., Miller, E. K., and Wang, X-J. (2007). A neural circuit model of flexible sensori-motor learning and forgetting on multiple time scales. Neuron 54, 319-333.

Fusi, S., Drew, P.J., and Abbott, L. F. (2005). Cascade models of synaptically stored memories. Neuron 45, 599-611.

Gallos, L. K., Song, C., Havlin, S., and Makse, H. A. (2007), Scaling theory of transport in complex biological networks. Proc. Natl. Acad. Sci. U.S.A. 104, 7746-7751.

Gammaitoni, L., Hanggi, P., Jung, P., and Marchesoni, F. (1998). Stochastic resonance. Rev. Mod. Phys. 70 223-287.

Gefen, Y., Mandelbrot, B. B., and Aharony, A. (1980). Critical phenomena on fractal lattices. Phys. Rev. Lett. 45 , 855-858.

Gerstein, G. L., and Mandelbrot, B. (1964). Random walk models for the spike activity of a single neuron. Biophys. J. 4, 41-68.
Gilboa, G., Chen, R, and Brenner, N. (2005). History-dependent multipletime-scale dynamics in a single-neuron model. J. Neurosci. 25, 6479-6489.

Gilden, D. J. (1997). Fluctuations in the time required for elementary decisions. Psychol. Sci. 8, 296-301.

Gilden, D. L. (2001). Cognitive emissions of 1/f noise. Psychol. Rev. 108, 33-56.

Gireesh, E. D., and Plenz, D. (2008). Neuronal avalanches organize as nested theta- and beta/gamma oscillations during development of cortical layer 2/3. Proc. Natl. Acad. Sci. U.S.A. 105, 7576-7581.

Gisiger, T. (2001). Scale invariance in biology: coincidence or footprint of a universal mechanism? Biol. Rev. 76 161-209.

Giugliano, M., Darbon, P., Arsiero, M., Luescher, H. R., and Streit, J. (2004). Single neuron discharge properties and network activity in dissociated cultures of neocortex. J. Neurophysiol. 92, 977-996.

Gong, P., Nikolaev, A. R., and Leeuwen, C. V. (2002). Scale-invariant fluctuations of the dynamical synchronization in human brain electrical activity. Neurosci. Lett. 336, 33-36.

Goychuk, I., and Hanggi, P. (2002). Ion channel gating: a first-passage time analysis of the Kramers type. Proc. Natl. Acad. Sci. U.S.A. 99, 3552-3556.

Greenlee, M. W., and Magnussen, S. (1987). Saturation of the tilt after effect. Vision res. 27, 1041-1043.

Griffin, L., West, D. J., and West, B. J. (2000). Random stride intervals with memory. J. Biol. Phys. 26, 185-2000.

Grigolini, P., Aquino, G., Bologna, M., Lukovic, M., and West, B. J. (2009). A theory of $1 / \mathrm{f}$ noise in human cognition. Physics A 388, 4192-4204.

Grinstein, G. (1995). “Generic scale invariance and self-organized criticality," in Scale Invariance, Interfaces and NonEquilibrium Dynamics, eds A. McKane, M. Droz, J. Vannimenus, and D. Wolf (New York: Plenum Press), 261-293.

Grüneis, F. (2001). 1/f Noise, Intermittency and Clustering Poisson Process. Fluct. Noise Lett. 1, R119-R130.

Grüneis, F., Nakao, M., Mizutani, Y., and Yamamoto, M, Meesmann, M., and Musha, T. (1993). Further study on $1 / f$ fluctuations observed in central single neurons during REM sleep. Biol. Cybern. 68, 193-198.

Guimera, R., Danon, L., Diaz-Guilera, F., Giralt, F., and Arenas, A. (2003). Self-similar community structures in a network of Human interactions. Phys.Rev. E 68, 065103R.

Hagmann, P., Cammoun, L., Gigandet, X., Meuli, R., Honey, C., Van Wedeen, J., and Spornsd, O. (2008). Mapping 
the structural core of human cerebral cortex. PLoS Biol. 6, e159. doi:10.1371/ journal.pbio.0060159.

Haken, H. (1983). Synergetics: An Introduction: Nonequilibrium Phase Transitions and Self-organization in Physics, Chemistry and Biology. New York: Springer.

Haken, H., Kelso, J. A., and Bunz, H. (1985). A theoretical model of phase transitions in human hand movements. Biol. Cybern. 51, 347-356.

Haldeman, C., and Beggs, J. M. (2005). Critical branching captures activity in living neural networks and maximizes the number of metastable states. Phys. Rev. Lett. 94, 058101.

Harris, K. D. (2005). Neural signatures of cell assembly organization. Nat. Rev. Neurosci. 6, 399-407.

Harris, T. E. (1989). The Theory of Branching Processes. New York: Dover.

Harrison, K. H., Hof, P. R., and Wang, S. S. -H. (2002). Scaling laws in the mammalian neocortex: does form provide clues to function? J. Neurocytol. 31, 289-298.

Hausdorff, J. M., and Peng, C. K. (1996). Multiscaled randomness: a possible source of $1 / \mathrm{f}$ noise in biology. Phys. Rev. E 54, 2154-2157.

Hausdorff, J. M., Peng, C. K., Ladin, Z., Wei, J. Y., and Goldberger, A. L. (1995). Is walking a random event? Evidence for long-range correlations in stride interval of human gait. J. Appl. Physiol. 78, 349-358.

He, B. J., Zempel, J. M., Snyder, A. Z., and Raichle, M. E. (2010). The temporal structures and functional significance of scale 0 -free brain activity. Neuron 66, 353-369.

Hennig, M. H., Adams, Ch., Willshaw, D., and Sernagor, E. (2009). Early-stage waves in the retinal network emerge close to a critical phase transition between local and global functional connectivity. J. Neurosci. 29, 1077-1086.

Henry, B. I., and Wearne, S. L. (2000). Fractional reaction-diffusion. Physica A 276, 448-455.

Herz, A. V., and Hopfield, J. J. (1995). Earthquake cycles and neural reverberations: collective oscillations in systems with pulse coupled threshold oscillators. Phys. Rev. Lett. 75, 1222-1225.

Hilgetag, C. C., and Kaiser, M. (2004). Clustered organization of cortical connectivity. Neuroinformatics 2, 353-360.

Hilgetag, C. C., Koetter, R., Stephan, K. E., and Sporns, O. (2002)."Computational methods for the analysis of brain connectivity," in Computational Anatomy, ed. G. A. Ascoli (Totowa, NJ: Humana Press), 295-335.
Honey, C. J., Kotter, R., Breakspear, M., and Sporns, O. (2007). Network structure of the cerebral cortex shapes functional connectivity on multiple time scales. PNAS, 104, 10240-10245.

Honey, C. J., Sporns, O., Cammoun, L., Thiran, J. P., Meuli, R., and Hagmann, P. (2009). Predicting human resting state functional connectivity from structural connectivity. Proc. Natl. Acad. Sci. U.S.A. 106, 2035-2040.

Hsu, K. J., and Hsu, A. (1991). Selfsimilarity of the "1/f noise" called music. Proc. Natl. Acad. Sci. U.S.A. 88, 3507-3509.

Hughes, B. D., Montroll, E. W., and Shlesinger, M. F. (1982). Fractal random walks. J. Stat. Phys. 28, 111-126.

Hütt, M-T., and Lesne,A. (2009). Interplay between topology and dynamics in excitation patterns on hierarchical graphs. Neuroinformatics 3, 1-10.

Huxley, J. S. (1932). Problems of Relative Growth. New York: The Dial Press.

Iannacone, P. M., and Khokha, M. (1995). Fractal Geometry in Biological Systems. Boca Raton, FL: CRC Press.

Im, K., Lee, J. M., Kim, S. H., Lyttelton, K. S. H., Evans, A. C., and Kim, S. L. (2008). Brain size and cortical structure. Cereb. Cortex 18, 2181-2191.

Jantzen, K. J., Steinberg, F. L., and Kelso, J. A. S. (2008). Coordination dynamics of large scale neural circuitry underlying rhythmic sensorimotor behavior. J. Cogn. Neurosci. 21, 2420-2433.

Jeffrey, H. J. (1990). Chaos game representation of gene structure. Nucleic Acid Res. 18, 2163-2170.

Jelinek, H. F., and Elston, G. N. (2001). Pyramidal neurones in macaque visual cortex: interareal phenotypic variation of dendritic branching patterns. Fractals 9, 287-295.

Jelinek, H. F., Elston, N., and Zietsch, B. (1995). Fractal analysis: pitfalls and revelations in neuroscience," in Fractals in Biology and Medicine, eds G.A. Losa, D. Merlini, T. F. Nonnenmacher, and E. R. Weibel (Basel: Birkhauser) 169-187.

Jones, C. L., and Jelinek, H. F. (2001). Wavelet packet fractal analysis of neuronal morphology. Methods 24, 347358. doi: 10.1006/meth.2001.1205.

Juanico, D. E., Monterola, C., and Saloma, C. (2007). Self-organized critical branching in systems that violate conservation laws. New J. Phys. 9, 1-17.

Kadanoff, L. P. (1990). Scaling and universality in statistical Physics. Physica A 163, 1-14.

Kadanoff, L. P., Nagel, S. R., Wu, L., and Zhou, S. (1989). Scaling and universality on avalanches. Physical Review A 39, 6524-6537.
Kaiser, M. (2007). Brain architecture: design for natural computation. Phil. Trans. R. Soc. 365, 3033-3045.

Kaiser, M., Goerner, M., and Hilgetag, C. C. (2007). Criticality and spreading dynamics in hierarchical cluster networks without inhibition. New J. Physics 9, 110-123.

Kaiser, M., and Hilgetag, C. C. (2010) Optimal hierarchical modular topologies for producing limited sustained activation of neural networks. Front. Neuroinform. 4, 8. doi:10.3389/ fninf.2010.00008.

Kapellou, O., Counsell, S. J., and Kennea, N. (2006). Abnormal cortical development after premature birth shown by altered allometric scaling of brain growth. PLoS Med. 3, e265. doi: 10.1371/journal.pmed.0030265.

Karperien, A. L., and Jelinek, H. F. (2008). Box-counting analysis of microglia form in schizophrenia, Alzheimer's disease, and affective disorder. Fractals 16, 103-107.

Kass, R. E., and Ventura, V. (2001). A spike-train probability model. Neural. Comput. 13, 1713-1720.

Katsaloulis, P., and Verganelakis, D. A. (2009). Fractal dimension and lacunarity of tractography images of the human brain. Fractals 17, 181-189.

Kaulakys, B., and Meskauskas, T. (1998) Modeling 1/f noise. Phys. Rev. E, 58, 7013-7019.

Kaulakys, B., Ruscekas, J., Gontis, V., and Alaburda, M. (2006). Nonlinear stochastic models of $1 / \mathrm{f}$ noise and power-law distributions. Physica A 365, 217-221.

Kello, C. T., Beltz, B. C., Holden, J. G., and Van Orden, G. C. (2007). The emergent coordination of cognitive function. J. Exp. Psychol. 136, 551-568.

Kelso, J.A.S. (1984). Phase transitions and critical behavior in human bimanual coordination. Am. J. Physiol. Regul. Integr. Comp. 15, R1000-R1004.

Kelso, J. A. S. (1995). Dynamic Patterns: The Self Organization of Brain and Behavior. Cambridge, MA: MIT Press.

Kelso, J. A. S. (2010). Instabilities and phase transitions in human brain and behavior. Front. Hum. Neurosci. 4, 1-2. doi:10.3389/fnhum.2010.00023.

Kelso, J. A. S., Bressler, S. L., Buchanan, S., DeGuzman, G. C., Ding, M., and Fuchs, A. (1992). A phase transition in human brain and behavior. Phys. Lett. A 169, 134-144.

Kelso, J.A.S., and Engstrøm,D. (2006). The Complementary Nature. Cambridge, MA: MIT Press.

Kelso, J. A. S., and Tognoli, E. (2007). "Toward a complementary neuroscience: metastable coordination dynamics of the brain," in Neurodynamics of Cognition and Consciousness, eds R. Kozma and L. Perlovsky (Berlin: Springer), 39-59.

Kim, J. S., Goh, K. I., Kahng, B., and Kim, D. (2007). Fractality and self-similarity in scale-free networks. New J. Phys. 9, 177.

King, C. C. (1991). Fractal and chaotic dynamics in the nervous system. Progr. Neurobiol. 36, 279-308.

Kinouchi, O., and Copelli, C. (2006). Optimal dynamical range of excitable networks at criticality. Nat. Phys. 2, 348-352.

Kinouchi, O., and Prado, C. P. C. (1999). Robustness of scale invariance in models with self-organized criticality. Phys. Rev. E 59, 4964-4969.

Kitzbichler, M. G., Smith, M. L., Christensen, S. R., and Bullmore, E. (2009). Broadband criticality of human brain network synchronization. PLoS Comput. Biol. 5, e1000314. doi:10.1371/journal.pcbi.1000314.

Kleinz, M., and Osler, T. J. (2000). A child's garden of fractional derivatives. College Math. J. 31, 82-88.

Kniffki, K.-D., Pawlak, M., and VahleHinz, C. (1993). Scaling behavior of the dendritic branches of thalamic neurons. Fractals 1, 171-178.

Kniffki, K.-D., Pawlak, M., Vahle-Hinz, C. (1994). "Fractal dimension and dendritic branching of neurons in the soimatosensory thalamus," in Fractals in Biology and Medicine, eds T. F. Nonnenmacher, G. Losa, and E. R. Weibel (Basel: Birkhauser Verlag), 221-229.

Kodama, T., Mushiake, H., Shima, K., Nakahama, H., and Yamamoto, M. (1989). Slow fluctuations of single unit activities of hippocampal and thalamic neurons in cats. I. relation to natural sleep and alert states. Brain Res. 487, 26-34.

Kolen, J. F. (1994). Fool's gold: extracting finite state machines from recurrent network dynamics. Adv. Neur. Inform. Proc. Syst. 6, 501-508.

Kolen, J. F. (2006). "Dynamical systems and iterated function systems," in A Field Guide to Dynamical Recurrent Networks, ed. J. F. Kolen and S. C. Kremer (New York: IEEE Press Inc.), 57-81.

Korn, H., and Faure, P. (2003). Is there chaos in the brain? II. Experimental evidence and related models. CR Biol. 326, 787-840.

Koulakov, A. A. (2010). On the scaling law for cortial magnification factor. arXiv:1002.4368v1[q-biol.NC].

Koutsoyiannis, D. (2002). The Hurst phenomenon and fractional Gaussian noise made easy. Hydrol. Sci. 47, 573-595. 
Kozma, R., Puljic, M., Ballister, P., Bollobas, B., and Freeman, W. J. (2005). Phase transitions in the neuropercolation model of neural populations with mixed local and non-local interactions. Biol. Cybern. 92, 367-379.

Kuikka, J., and Tiihonen, J. (1998). Fractal analysis - a new approach to receptor imaging. Ann. Med. 30, 242-248.

Kulish, V., Sourin, A., and Sourina, O. (2006). Human electroencephalograms seen as fractal time series: mathematical analysis and visualization. Comput. Biol. Med. 36, 291-302.

Kuramoto, Y. (1984). Chemical Oscillations, Waves and Turbulence. Berlin: Springer.

LaBarbera, M. (1989). Analyzing body size as a factor in ecology and evolution. Annu. Rev. Ecol. Syst. 20, 97-117.

Lakatos, P., Karmos, G., Mehta, A. D., Ulbert, I., and Schroeder, C. E. (2008). Entrainment of neuronal oscillations as a mechanism of attentional selection. Science 320, 110-113.

Lakatos, P., Shah, A. S., Knuth, K. H., Ulbert, I., Karmos, G., and Schroeder, C. E. (2005). An oscillatory hierarchy controlling neuronal excitability and stimulus processing in the auditory cortex. J. Neurophysiol. 94, 1904-1911.

Lambiotte, R. (2010). Multi-scale modularity in complex networks. arXiv:1004.4268v1 [physics.soc-ph].

Lambiotte, R., Delvenne, J-C., and Barahona,M.(2009).Laplacian dynamics and multiscale modular structure in networks. arXiv:0812.1770v3 [physics.soc-ph].

Laughlin, R. B. (2005). A Different Universe: Reinventing Physics from the Bottom Down. New York: Basic Books.

Lenaerts, T., Chu, D., and Watson, R. (2005). Dynamical hierarchies. Artif. Life 11, 403-405.

Le Van Quyen, M. (2003). Disentangling the dynamic core: a research program for a neurodynamics at the large scale. Biol. Res. 36, 67-88.

Levina, A., Herrmann, J. M., and Geisel, T. (2007). Dynamical synapses causing self-organized criticality in neural networks. Nat. Phys. 3, 857-860

Levina, A., Herrmann, J. M., and Geisel, T. (2009). Phase transition towards criticality in a neural system with adaptive interactions. Phys. Rev. Lett. 102,118110 .

Levy, P. (1954). Theorie de L'addition des Variables Aleatoires. Paris: GauthierVillars.

Levy, S. D., and Pollack, J. B. (2002). Logical computation on a fractal neural substrate. http://www.demo.cs.brandeis. edu/papers/raam-ijcnn01.ps.gz.
Lewis, C. D., Gebber, G. L., Larsen, P. D., and Barman, S. M. (2001). Longterm correlations in the spike trains of Medullary sympathetic neurons. J. Neurophysiol. 85, 1614-1622.

Li, M. (2010). Fractal time series - a tutorial review. Math. Probl. Eng. Article ID 157264.

Liebovitch, L. S., Fischbarg, J., Koniarek, J. P., Todorova, I., and Wang, M. (1987). Fractal model of ion-channel kinetics. Biochim. Biophys. Acta 896, 173-180.

Liebovitch, L. S., Scheurle, D., Rusek, M., and Zochowski, M. (2001). Fractal methods to analyze ion channel kinetics. Methods 24, 359-275.

Linkenkaer-Hansen, K. (2003). Scaling and criticality in large-scale neuronal activity. Lect. Notes Phys. 621, 324-338.

Linkenkaer-Hansen, K., Nikouline, V. V., Palva, J. M., and Ilmoniemi, R. J. (2001). Long-range temporal correlations and scaling behavior in human brain oscillations. J. Neurosci. 21, 1370-1377.

Linkenkaer-Hansen, K., Nikulin, V. V., Palva, J. M., Kaila, K., and Ilmoniemi, R.J. (2004a). Stimulus-induces change in long-range temporal correlations and scaling behaviour of sensorimotor oscillations. Eur. J. Neurosci. 19, 203-211.

Linkenkaer-Hansen, K. Nikuli, V. V. Palva, S., Ilmoniemi, R. J., and Palva, J. M. (2004b). Prestimulus oscillations enhance psychophysical performance in Humans. J. Neurosci. 24, 10186-10190.

Logothetis, N. K. (2002). The neural basis of the blood-oxygen-level-dependent functional magnetic resonance imaging signals. Philos. Trans. R. Soc. London B Biol. Sci. 357, 1003-1037.

Lowen, S. B., Cash, S. S., Poo, M.-M., and Teich, M. C. (1997). Quantal neurotransmitter secretion rate exhibits fractal behavior. J. Neurosci. 17, 5666-5677.

Lowen, S. B., Liebovitch, L. S., and White, J. A. (1999). Fractal ion-channel behavior generates fractal firing patterns in neuronal models. Phys. Rev. E, 59, 5970-5980.

Lowen, S. B., and Teich, M. C. (1993). Fractal auditory nerve firing patterns may derive from fractal switching in sensory hair cell ion channels. AIP Conf. Proceedings 285, eds P. H. Handel, A. L. Chung, American Institute of Physics. pp. 745-748.

Lowen, S. B., and Teich, M. C. (2005). Fractal based Point Processes. New York: Wiley.

Lundstrom, B. N., Higgs, M. H., Spain, W. J., and Fairhall, A. (2008). Fractional differentiation by neoircortical pyramidal neurons. Nat. Neurosci. doi: 10.1038/nn.2212.

Maess, B., Koelsch, S., Gunter, T. C., and Friederici,A. D. (2001). Musical syntax is processed in Broca's area: an MEG study. Nat. Neurosci. 4, 540-545.

Magin, R. L. (2006). Fractional Calculus in Bioengineering. Redding, CT: Begell House Publishers, Co.

Magnussen, S., and Greenlee, M. W. (1985). Marathon adaptation to spatial contrast: saturation in sight. Vision Res. 25, 1409-1411.

Maimon, G., and Assad, J. A. (2009). Beyond Poisson: increased spike-time regularity across primate parietal cortex. Neuron 62, 426-440.

Mandelbrot, B. B. (1977). The Fractal Geometry of Nature. New York: W. H. Freeman and Co.

Mandelbrot, B. B., and van Ness, J. W. (1968). Fractional Brownian motions, fractional noises and applications. SIAM Rev. 10, 422-437.

Marro, J., and Dickman, R. (1999). Nonequilibrium Phase Transitions in Lattice Models. Cambridge, UK Cambridge University Press.

Maxim, V., Sendur, L., Fadili,J., Suckling, J., Gould, R., Howard, R., and Bullmore, E. (2005). Fractional Gaussian noise, functional MRI and Alzheimer's disease. Neuroimage 25, 141-158.

Mazzoni, A., Broccard, F. D., Garcia-Perez, E., Bonifazi, P., Ruaro, M.E., and Torre, V. (2007). On the dynamics of the spontaneous activity in neuronal networks. PLoS ONE 5, e349. doi:10.1371/ journal.pone.0000439.

McKenna, T. M. (1992). Single Neuron Computation. New York: Elsevier.

Mayer-Kress, G., and Layne, S. P. (1987). Dimensionality of the Human Electroencephalogram. Ann. N.Y. Acad. Sci. 504, 62-87.

Meunier, D., Lambiotte, R., Fornito, A., Ersche, K. D., and Bullmote, E. T. (2009). Hierarchical modularity in human brain functional networks. Front. Neuroinformatics 3: Article 37 doi:10.3389/neuro.11.037.2009.

Meyer-Lindenberg, A., Ziemann, U., Hajak, G., Cohen, L., and Berman, K. F. (2002). Transition between dynamical states of differing stability in the human brain. Proc. Natl. Acad. Sci. U.S.A. 99, 10948-10953.

Miller, S. L., Miller,W.M., and McWhorter, P. J. (1993). Extremal dynamics: a unifying physical explanation of fractals, $1 / \mathrm{f}$ noise and activated processes. $J$. Appl. Phys. 73, 2617-2628.

Millhauser, G. L., Salpeter, E. E., and Oswald, R.E. (1988). Diffusion models of ion-channel gating and the origin of power-law distributions from singlechannel recording. Proc. Natl. Acad. Sci. U.S.A. 85, 1503-1507.
Millotti,E. (2002). 1/f noise: a pedagogical review. arXiv:physics/0204033v1.

Milosevic, N. T., Ristanovic, D., and Stankovic, J. B. (2005). Fractal analysis of laminar organization in spinal cord. J. Neurosci. Methods 146, 198-204. doi: $10.1016 / \mathrm{j}$. neumeth.2005.02.009.

Milosevic, N. T., Ristanovic, D., Stankovic, J. B., and Gudovic, R. (2007). Fractal analysis of dendritic arborisation patterns of stalked and islet neurons in substantia gelatinosa of different species. Fractals 15, 1-6.

Milstein, J., Mormann, F., Fried, I., and Koch, C. (2009). Neural shot noise and Brownian 1/fbehavior in the local field potential. PLoS ONE 4, e4338. doi:10.1371/journal.pone.0004338.

Mitzenmacher, M. (2003). A brief history of generative models for power-law and lognormal distributions. Internet Math. 1, 226-251.

Monto, S., Palva, S., Voipio, J., and Palva, J. M. (2008). Very slow EEG fluctuations predict the dynamics of stimulus detection and oscillation amplitudes in Humans. J. Neurosci. 28, 8268-8272.

Montroll, E. W., and West, B. J. (1979). "An enriched collection of stochastic processes," in Fluctuation Phenomena, eds E. W. Montroll and J. Lebowitz (Amsterdam, NY: North Holland), 61-175.

Montroll, E. W., and Weiss, G. (1965). Random walks on lattices II. J. Math Phys. 6, 167-182.

Morariu,V.V.,Cosa, A., Chis, M.A., Isvoran, A., and Morariu, L.-C. (2001). Scaling in cognition. Fractals 9, 379-391.

Mucha, P. J., Richardson, T., Macon, K., and Porter, M.A. (2009). Community structure in time-dependent, multiscale and multiplex networks. arXiv:0911.1824v1 [physics.data-an].

Müller-Linow, M., Hilgetag, C. C., and Huett, M-T. (2008). Organization of excitable dynamics in hierarchical biological networks. PLoS Comput. Biol. 4, e1000190. doi:10.1371/journal. pcbi. 1000190 .

Muzy, J. F., Bacry, E., and Arneodo, A. (1993). Multifractal formalism for fractal signals: the structure-function approach versus wavelet transform modulus maxima methods. Phys. Rev. E 47, 875-884.

Nakanishi, K., and Kukita, F. (1998). Functional synapses in synchronized bursting of neocortical neurons in culture. Brain Res. 795, 137-146.

Newman, M. E. L. (1996). Self-organized criticality, evolution and the fossil extinction record. Proc. R. Soc. Lond., B, Biol. Sci. 263, 785-792.

Newman, M. E. J. (2005). Power-laws, Pareto distributions and Zipf's law. Contemp. Phys. 46, 323-351. 
Newman, M. E. J., and Girvan, M. (2004). Finding sand evaluating community structure in networks. Phys. Rev. E. 69, 026113.

Nikulin, V.V., and Brismar, T. (2005). Longrange temporal correlations in electroencephalographic oscillations: relation to topography, frequency band, age and gender. Neuroscience 130, 549-558.

Northoff, G., Heinzel, A., de Greck, M., Bermpohl, F., Dobrowolny, H., and Panksepp, J. (2006). Self-referential processing in our brain - a metaanalysis of imaging studies on the self. Neuroimage 31, 440-457,

Novikov, E., Novikov, A., ShannahoffKhalsa, D., Schwartz, B., and Wright, J. (1997). Scale-similar activity in the brain. Phys. Rev. E, 56, R2387.

Ohlshausen, B. A., and Field, D. J. (1997). Sparse coding with an over complete basic set: a strategy employed by V1? Vision Res. 37, 3311-3325.

Orer, H. S., Das, M., Barman, S. M., and Gebber, G. L. (2003). Fractal activity generated independently by medullary sympathetic premotor and preanglionic sympathetic neurons. $J$. Neurophysiol. 90, 47-54.

Paczuki, M., Maslov, S., and Bak, P. (1996). Avalanche dynamics in evolution, growth and depinning models. Phys. Rev. E 53, 414-443.

Pajevic, S., and Plenz, D. (2009). Efficient network reconstruction from dynamical cascades identifies small world topology of neuronal avalanches. PLoS Comput. Biol. 5, e1000271. doi:10.1371/journal. pcbi. 1000271.

Palva, J. M., Palva, S., and Kaila, K. (2005). Phase synchrony among neuron oscillations in the human cortex. J. Neurosci. 25, 3962-3972.

Papa, A. R. R., and da Silva, L. (1997). Earthquakes in the brain. Theory Biosci. 116, 321-327.

Paramanathan, P., and Uthayakumar, R. (2008). Application of fractal theory in analysis of human electroencephalographic signals. Comput. Biol. Med. 38, 372-378.

Patel, A. D. (2003). Language, music, syntax, and the brain. Nat. Neurosci. 6, 674-681.

Pattee, H. H. (2001). The physics of symbols: bridging the epistemic cut. BioSystems 60, 5-21.

Park, J. P., and Newman, M. E. J. (2004). Statistical mechanics of networks. Phys. Rev. E, 70, 066117.

Pasquale, V. P., Massobrio, P., Bologna, L. L., Chiappalone, M., and Martinoia, S. (2008).Self-organization and neuronal avalanches in networks of dissociated cortical neurons. Neuroscience 153, 1354-1369.

Pellionisz, A. J. (1989). "Neural geometry: towards a fractal model of neurons," in Models of Brain Function, ed. R. M. L. Cotterill (Cambridge: Cambridge University Press) 1-9.

Penrose, O. (1986). Phase transitions on fractal lattices with long-range interactions. J. Stat. Phys. 45, 69-88.

Perez-Mercader, J. (2004). "Coarsegraining, scaling and hierarchies," in Nonextensive Entropy-interdisciplinary Applications, eds M. Gell-Mann and C. Tsallis (Oxford University Press), 357-376.

Perkel, D. H., and Feldman, M.W. (1979). Neurotransmitter release statistics: moment estimates for inhomogeneous Bernoulli trials. J. Math. Biol. 7, 31-40.

Petermann, T., Thiagarajan, T. C., Lebedev, M. A., Nicolelis, M. A. L., Chialvo, D. R., and Plenz, D. (2009). Spontaneous cortical activity in awake monkeys composed of neuronal avalanches. Proc. Natl. Acad. Sci. U.S.A. 106, 15921-15926.

Plenz, D., and Aertsen, A. (1996). Neuronal dynamics in cortex-striatum cultures II. Spatio-temporal characteristics of neuronal activity. Neuroscience 70, 893-924.

Plenz, D., and Chialvo, D. R. (2010). Scaling properties of neuronal avalanches are consistent with critical dynamics. arXiv:0912.5369v1 [q-bio. NC].

Plenz, D., and Thiagarajan, T. C. (2007). The organizing principles of neuronal avalanches: cell assemblies in the cortex? Trends Neurosci. 30, 101-110.

Podlubny, I. (1999). Fractional Differential Equations. San Diego, Academic Press.

Poli, S.-S., Ooyen, A. V., and LinkenkaerHansen, K. (2008). Avalanche dynamics of human brain oscillations: relation to critical branching processes and temporal correlations. Hum. Brain Mapp. 29, 770-777.

Pollack, J. B. (1991). Induction of dynamical recognizers. Mach. Learn. 7, 227-252.

Press, W. H. (1978). Flicker noises in astronomy and elsewhere. Comments Astrophys. 7, 103-119.

Reynolds, A. M. (2009). Scale-free animal movement patterns: Levy walks outperform fractional Brownian motions and fractional Levy motions in random search scenarios. J. Phys. A 42, 434006.

Richmond, B. J., Optican, L. M., and Spitzer, H. (1990). Temporal encoding of two-dimensional patterns by single units in primary visual cortex I. Stimulus-Response relations. J. Neurophysiol. 64, 351.

Rikvold, P. A., Kornoiss, G., White, C. J., Novotny, M. A., and Sides, S. W. (1999). arXiv:cond-mat/9904028v2 [cond-mat.stat.mech].
Robinson, H. P. C., Kawahara, M., Jimbo, Y., Torimitsu, K., Kuroda, Y., and Kawana, A. (1993). Periodic synchronized bursting and intracellular calcium transients elicited by low magnesium in cultured cortical neurons. J. Neurophysiol. 70 1606-1616.

Rodriguez, M., Pereda, E., Gonzalez, J. Abdala, P., and Obeso, J. A. (2003). Neuronal activity in the substantia nigra in the anesthetized rat has fractal characteristics: evidence for firing code patterns in the basal ganglia. Exp. Brain Res. 151, 167-172.

Roerig, B., Chen, B. (2002). Relationships of local inhibitory and excitatory circuits to orientation preference maps in Ferret visual cortex. Cereb. Cortex 12, 187-198.

Roncaglia, R., Mannella, R., and Grigolini, P. (1994). Fractal properties of ion channels and diffusion. Math. Biosci. $123,77-101$.

Rose, D., and Lowe, I. (1982). Dynamics of adaptation to contrast. Perception 11, 505-528.

Rotshenker, S., and Rahamimoff, R. (1970). Neuromuscular synapse: stochastic properties of spontaneous release of transmitter. Science 170 648-649.

Ruseckas, J., and Kaulakys, B. (2010). 1/f noise from nonlinear stochastic differential equations. arXiv:1002.4316v1 [nlin.AO].

Sakai, Y., Funahashi, S., and Shinomoto, S. (1999). Temporally correlated inputs to leaky integrate-and-fire models can reproduce spiking statistics of cortical neurons. Neural Networks, 12, 1181-1190.

Salinas, E., and Sejnowski, T. J. (2002) Integrate-and-fire neurons driven by correlated stochastic input. Neural. Comput. 14, 2111-2155.

Sales-Pardo, M., Guimera, R., Moreira, A. A., and Amaral, L. M. A. (2007). Extracting the hierarchical organization of complex systems. Proc. Natl. Acad. Sci. USA 104, 15224-15229.

Salvadori, G., and Biella, G. (1994). "Discriminating properties of wide dynamic range neurons my means of universal multifractals," in Fractals in Biology and Medicine II, eds G. A. Losa, D. Merlini, T.F. Nonnenmacher, and E. R. Weibel (Basel: Birkhauser-Verlag), 314-325.

Scafetta, N., Marchi, D., and West, B. J. (2009). Understanding the complexity of human gait dynamics. Chaos 19, 026108.

Scheffer, M., Bascompte, J., Brock, W. A., Brovkin, V., Carpenter, S. R., Dakos, V., Held, H., van Nes, E. H., Rietkerk, M. D., and Sugihara, G. (2009). Earlywarning signals for critical conditions. Nature 461, 53-59.
Schierwagen, A. (2008). Neuronal morphology: shape characteristics and models. Neurophysiology 40, 310-315.

Segev, R., Baruchi, I., Hulata, E., and BenJacob, E. (2004). Hidden neuronal correlations in cultured networks. Phys. Rev. Lett. 92, 118102.

Segev, R., Benveniste, M., Hulata, E., Cohen, N., Palevski, A., Kapon, E., Shapira, Y., and Ben-Jacob, E. (2002). Long behavior of lithographically prepared in vitro neuronal networks. Phys. Rev. Lett. 88, 118102.

Seuront, L. (2010). Fractals and Multifractals in Ecology and Aquatic Science. Boca Raton: CRC Press.

Shadlen, M. N., and Newsome, W. T. (1998). The variable discharge of cortical neurons: implications for connectivity, computation, and information coding. J. Neurosci. 18, 3870-3896.

Shahverdian, A. Y., and Apkarian, A. V. (1999). On irregular behavior of neuron spike trains. Fractals 7, 93-103.

Shalizi, C. R., Camperi, M. F., and Klinkner, K. L. (2006). Discovering functional communities in dynamical networks. arXiv:q-biol/0609008v2 [q-bio.NC].

Shew, W. L., Yang, H., Petermann, T., Roy, R., and Plenz, D. (2009). Neuronal avalanches imply maximum dynamic range in cortical networks at criticality. J. Neurosci. 29, 15600-15595.

Shimizu, Y., Barth, M., Windischberger, C., Moser, E., and Thurner, S. (2004). Wavelet-based multifractal analysis of fMRI time series. Neuroimage 22, 1195-1202.

Shimono, M., Owaki, T., Aman, K., Kitajo, K., and Takeda, T. (2007). Functional modulation of power-law distribution in visual perception. Phys. Rev. E, 75, 051902 .

Shinomoto, S., Shima, K., and Tanji, J. (2003). Differences in spiking patterns among cortical neurons. Neural. Comput. 15, 2823-2842.

Shlesinger, M. F., West, B. J., and Klafter, J. (1987). Levy dynamics of enhanced diffusion: application to turbulence. Phys. Rev. Lett. 58, 1100-1103.

Simon, H. A. (1955). On a class of skew distribution functions. Biometrika 42 , 425-440.

Simon, H. A. (1962). The architecture of complexity. Proc. Amer. Phil. Soc. 106, 467-482.

Simon, H.A. (1973). "The organization of complex systems," in Hierarchy Theory, ed. H. H. Pattee (NY: Brazillier), 3-27.

Simon, H. A. (1996). The Sciences of the Artificial, 3rd Edn. Cambridge, MA: MIT Press.

Smith, T. G., Jr., Marks, W. B., Lange, G. D., Sheriff, W. H., Jr., and Neale, 
E. A. (1989). A fractal analysis of cell images. J. Neurosci. Methods 27, 173-180.

Smolders, F. D. J., and Folgering, H. Th. M. (1977). Actions and Interactions of $\mathrm{CO}_{2}$ and $\mathrm{O}_{2}$ on the Controlling System of the Lung Ventilation. Ph.D. thesis, Nijmegen University, Netherlands.

Sokal,A., and Bricmont,J. (2004). "Defense of a modest scientific realism," in Knowledge and the World: Beyond the Science Wars. eds M. Carroer, J. Roggenhofer, G. Kueppers, and P. Banchard (New York: Springer)

Sokolov, I. M., Klafter, J., and Blumen, A. (2002). Fractional Kinetics. Phys. Today 48-54.

Soltys, Z., Ziaja, M., Pawlinski, R., Setkowicz,Z., and Janeczko, K. (2001). Morphology of reactive microglia in the injured cerebral cortex. Fractal analysis and complementary quantitative methods. J. Neurosci. Res. 63, 90-97.

Song, C., Havlin, S., and Makse, H. A. (2005). Self-similarity of complex networks. Nature 433, 392-395.

Song, C., Havlin, S., and Makse, H. A. (2006). Origins of fractality in the growth of complex networks. Nat. Phys. 2, 275-281.

Sornette, D. (2000). Critical Phenomena in Natural Sciences. Berlin: Springer.

Sporns, O. (2006). Small-world connectivity, motif composition, and complexity of fractal neuronal composition. BioSystems 85, 55-64.

Sporns, O., Chialvo, D. R., Kaiser, M., and Hilgetag, C. C. (2004). Organization, development and function of complex brain networks. Trends Cog. Sci. 8, 418-425.

Sporns, O., Honey, C. J., and Koetter, R. (2007). Identification and classification of hubs in brain networks. PLoS One 10: e1049. doi:10.1371/journal. pone. 0001049 .

Sporns, O., and Koetter, R. (2004). Motifs in brain networks. PLoS Biol. 2, e369. doi:10.1371/journal.pbio.0020369.

Sporns, O., and Zwi, J. D. (2004). The small world of the cerebral cortex. Neuroinformatics 2, 145-162.

Stam, C. J. (2004). Functional connectivity patterns of human magnetoencephalographic recordings: a 'small-world' network? Neurosci. Lett. 355, 25-28.

Stam, C. J. (2005). Nonlinear dynamical analysis of EE and MEG: review of an emerging field. Clin. Neurophys. 116, 2266-2301.

Stam, C. J., and de Bruin, E. A. (2004). Scale-free dynamics of global functional connectivity in the human brain. Hum. Brain Mapp. 22, 97-109.

Stam, C. J., and Reijneveld, J. C. (2007). Graph theoretical analysis of complex network in the brain. Nonlinear Biomed. Phys, 1, 3 .
Stanley, H.E. (1987). Introduction to Phase Transitions and Critical Phenomena. Oxford, UK: Oxford University Press.

Stanley, H. E. (1999). Scaling, universality and renormalization: the three pillars of modern critical Phenomena. Rev. Mod. Phys. 71, S358-S366.

Stauffer, D., and Aharony, A. (1991/1994). Introduction to Percolation Theory. Boca Raton: CRC Press.

Stephen, D. G., and Dixon, J. A. (2009) The self-organization of insight: entropy and power-laws in problem solving. J. Problem Solving 2, 72-101.

Stevens, S.S. (1957). On the psychophysical law. Psychol. Rev. 64, 153-181.

Stevens, C. F., and Zador, A. M. (1998). Input synchrony and the irregular firing of cortical neurons. Nat. Neurosci. 1, 210-217.

Stewart, C. V., and Plenz, D. (2006) Inverted $U$ profile of dopamineNMDA mediated spontaneous avalanche recurrence in superficial layers o rat prefrontal cortex. J. Neurosci. 23, 8148-8159.

Stinchcombe, R. B. (1989). Fractals, phase transitions, and criticality. Proc. R. Soc. Lond. A, 423, 17-33.

Stoop, R., and Wagner, C. (2007). Neocortex's architecture optimizes computation, information transfer and synchronizability, at given total connection length. Int. J. Bifurc. Chaos $17,2257-2279$.

Suckling, J., Wink, A. M., Bernard, F. A., Barnes, A., and Bullmore, E. (2008). Endogenous multifractal brain dynamics are modulated by age cholinergic blockade and cognitive performance. J. Neurosci. Methods 174, 292-300.

Tabor, W. (2000). Fractal encoding of context-free grammars in connectionist networks. Expert Syst. 17, 41-56.

Takeda, T., Sakata, A., and Matsuoka, T. (1999). Fractal dimensions in the occurrence of miniature end-plate potentialin a vertebrate neuromuscular junction. Prog. Neuropsychopharmacol. Biol. Psychiatr. 23, 1157-1169.

Tateno, T., Kawana, A., and Jimbo, Y (2002). Analytical characterization of spontaneous firing in networks of developing rat cultured cortical neurons. Phys. Rev. E 65, 051924.

Tebbens, S. F., and Burroughs, S. M (2003). Self-similar criticality. Fractals 11, 221-231.

Teich, M. C., and Saleh, B. E. A. (1981) Interevent-time statistics for shotnoise-driven self-exciting point processes in photon detection. J. Opt. Soc. Am. 71, 771-776.

Teich, M. C., Johnson, D. H., Kumar, A. R., and Turcott, R. G. (1990). Rate fluctuations and fractional power-law noise recorded from cells in the lower auditory pathway of the cat. Hear. Res 46, 41-52.

Teich, M. C., Heneghan, C., Lowen, S. B., Ozaki, T., and Kaplan, E. (1997). Fractal character of the neural spike train in the visual system of the cat. $J$ Opt. Soc. Am. 14, 529-546.

Teramae, J.-N., and Fukai, T. (2007). Local cortical circuit model inferred from power-law distributed neuronal avalanches. J. Comput. Neurosci. 22, 301-312.

Thatcher, R. W., North, D. M., and Biver C. J. (2009). Self-organized criticality and the development of EEG phase reset. Hum. Brain Mapp. 30 553-574.

Thiagarajan, T. C., Lebedev, M. A. Nicolelis, M. A., and Plenz, D. (2010). Coherence potentials: loss-less, all-ornone network events in the cortex. PLoS Biol. 8, e1000278. doi:10.1371/ journal.pbio. 1000278 .

Thorson, J., and Biederman-Thorson, M (1974). Distributed relaxation processes in sensory adaptation. Science $183,161-183$.

Thurner, S., Lowen, S. B., Feurstein, M. C., Heneghan, C., Feichtinger, H. G. and Teich, M. C. (1997). Analysis, synthesis, and estimation of fractal-rate stochastic point processes. Fractals 5 , 565-595.

Thurner, S., Windischberger, C., Moser, E., Walla, P., and Barth, M. (2003). Scaling laws and persistence in human brain activity. Physica A 326, 511-521.

Tino, P. (1999). Spatial representation of symbolic sequences through iterative function systems. IEEE Trans. Systems, Man Cybernet. 29, 386-393.

Tognoli, E., and Kelso, J.A.S. (2009). Brain coordination dynamics: true and false faces of phase synchrony and metastability. Progr. Neurobiol. 87, 31-40.

Toib, A., Lyakhov, V., and Marom, S. (1998). Interaction between duration of activity and time course of recovery from slow inactivation in mammalian brain $\mathrm{Na}^{2+}$ channels. J. Neurosci. 18, 1893-1903.

Touboul, J., and Destexhe, A. (2009). Can power-law scaling and neuronal avalanches arise from stochastic dynamics? arXiv: 0910.0805v1.

Tsuda, I., and Kuroda, S. (2004). "A complex system approach to an interpretation of dynamic brain activity II. does Cantor coding provide a dynamical model for the formation of episodic memory?," in Cortical Dynamics, LNCS 3146, ed. P. Erdi (Berlin: Springer), 129-139.

Turcotte, D. L. (1999). Self-organized criticality. Rep. Prog. Phys. 62, 1377-1429.

Turing, A. M. (1950). Computing machinery and intelligence. Mind 59, 433-460.
Tsallis, C. (2009). Introduction to NonExtensive Statistical Mechanics: Approaching a Complex World. New York: Springer.

Tsallis, C., Levy, S. V. F., Souza, A. M. C. and Maynard, R. (1995). Statisticalmechanical foundation of the ubiquity of levy distributions in nature. Phys. Rev. Lett. 75, 3589-3593.

Uhlhaas, P. J., Pipa, G., Lima, B., Melloni, L., Neuenschwander, S., Nikolic, D., and Singer, W. (2009). Neural synchrony in cortical networks: history, concept and current status. Front. Integr. Neurosci. 3, 1-19. doi:10.3389/ neuro.07.017.2009.

Ulanovsky, N., Las, L., Farkas, D., and Nelken, I. (2004). Multiple time scales of adaptation in auditory cortex neurons. J. Neurosci. 17, 10440-10453.

Usher, M., and Stemmler, M. (1995). Dynamic pattern formation leads to 1/f noise in neural populations. Phys. Rev. Lett. 74, 326-329.

Usher, M., Stemmler, M., Koch, C., and Olami, Z. (1994). Network amplification of local fluctuations causes high spike rate variability, fractal firing patterns and oscillatory local field potentials. Neural. Comput. 6, 795-836.

van den Heuvel, M.P., Stam, C. J., Boersma, M., and Pol, H. E. H. (2008). Smallworld and scale-free organization of voxel-based resting-state functional connectivity in the human brain. Neuroimage 43, 528-539.

Van Orden, G. C., Holden, J. G., and Turvey, M. T. (2003). Self-organization of cognitive performance. J. Exp. Psych: Gen. 132, 331-350.

van Pelt, J., Coner, M. A., Wolters, P. S., Rutten, W. L. C., and Ramakers, G. J. A. (2004). Long-term stability and developmental changes in spontaneous network burst firing patterns in dissociated rat cerebral cortex cell cultures on microelectrode arrays. Neurosci. Lett. 361, 86-89.

van Vreeswijk, C. (2001). Information transmission with renewal neurons. Neurocomputing 38-40, 417-422.

Varanda, W. A., Liebovitch, L. S., Figueiroa, J. N., and Nogueira, R. A. (2000). Hurst analysis applies to the study of single calcium-activated potassium channel kinetics. J. Theor Biol. 206, 343-353.

Varela, F., Lachaux, J.-P., Rodriguez, E., and Martinerie, J. (2001). The Brainweb: phase synchronization and large scale integration. Nat. Rev. Neurosci. 2, 229-239.

Voss, R. F., and Clarke, J. (1975). '1/f noise' in music and speech. Nature 258, 317-318.

Wagenaar, D. A., Pine, J., and Potter, S. M. (2006a). An extremely rich repertoire of bursting patterns of bursting patterns during the development of 
cortical cultures. BMC Neuroscience 7, 7-11.

Wagenaar, D. A., Nadasky, Z., and Potter, S. M. (2006b). Persistent dynamic attractors in activity patterns of cultured neuronal networks. Phys. Rev. E 73, 051907.

Wagenmakers, E.-J.,Farrell, S., and Ratcliff, R. (2004). Estimation and interpretation of $1 /$ f $\alpha$ noise in human cognition. Psychon. Bull. Rev. 11, 579-615.

Wagenmakers, E.-J., Farrell, S., and Ratcliff, R. (2005). Human cognition and a pile of sand: a discussion on serial correlations and self-organized criticality. $J$. Exp. Psychol. Gen. 134, 108-116.

Wark, B., Lundstrom, B. N., and Fairhall, A. (2008). Sensory adaptation. Curr. Opin. Neurobiol. 17, 423-429.

Watts, D., and Strogatz, S. (1998). Collective dynamics of small world networks. Nature 393, 440-442.

Wen, Q., Stepanyants, A., Elston, G. N., Grosberg, A. Y., and Chklovskii, D. B. (2009). Maximization of the connectivity repertoire as a statistical principle governing the shapes of dendritic arbors. Proc. Natl. Acad. Sci. USA, 106, 12536-12541.

Werner, G., and Mountcastle, V. B. (1963). The variability of central neural activity in a sensory system, and its implications for the central reflection of sensory events. J. Neurophysiol. 26, 958-977.

Werner, G., and Mountcastle, V. B. (1964). Neural activity in mechanoreceptive cutaneous afferents; stimulus-response relations, Weber functions, and information transmission. J. Neurophysiol. 28, 359-394

Werner, G. (2007a). Perspectives on the neuroscience of cognitions and consciousness. BioSystems 87, 82-95.

Werner, G. (2007b). Metastability, criticality, and phase transitions in brain and its models. BioSystems 90, 496-508.

Werner, G. (2009a). Viewing brain processes and critical state transitions across levels of organization: neural events in cognition and consciousness, and general principles. BioSystems 96 , 114-119.

Werner, G. (2009b). Consciousness related neural events viewed as brain state space transitions. Cogn. Neurodyn. 3, 83-95.

Werner, G. (2009c). On critical state transitions between different levels in neu- ral systems. New Math. Nat. Comput. 5, 185-196.

West, B. J. (1999). Physiology, promiscuity and prophecy at the millenium: a tale of tails. Studies of Nonlinear Phenomena in the Life Sciences, Vol. 7. Singapore: World Scientific.

West, B. J. (2006). Where medicine went wrong: rediscovering the path to complexity. Singapore: World Scientific.

West, B. J. (2009). Control from an allometric perspective. Adv. Exp. Med. Biol. 629, 57-82.

West, B. J., Allegrini, P., and Grigolini, P. (1994). "Dynamical generators of levy statistics in biology," in Fractals in Biology and Medicine, Vol. II, eds G. A. Losa, D. Merlini, T. F. Nonnenmacher, and E. R. Weibel (Basel: Birkhauser), 169-187.

West, B. J., Bologna, M., and Grigolini, P. (2003). Physics of Fractal Operators. New York: Springer.

West, B.J., and Deering, W. (1994). Fractal physiology for physicists: levy statistics. Physics Reports 246, 1-100.

West, B. J., and Deering, B. (1995). The Lure of Modern Science. Studies in Nonlinear Phenomena in Life Sciences, Vol. 3. Singapore: World Scientific.

West, B. J., Geneston, E. L., and Grigolini, P. (2008). Maximizing information exchange between complex networks. Phys. Rep. 468, 1-99.

West, B. J., and Griffin, L. (1999). Allometric control, inverse powerlaws and human gait. Chaos Solitons Fractals 10, 1519-1527.

West, B. J., and Nonnenmacher, T. (2001). An ant in a gurge. Phys. Lett. A, 278, 255-259.

West, B. J., and Scafetta, N. (2003). Nonlinear dynamical model of human gait. Phys. Rev. E 67, 051917.

West, G. B., and Brown, J.H. (2004). Life's universal scaling laws. Phys. Today, 57, $36-42$.

West, G. B., Grigolini, P., Metzler, R., and Nonnenmacher, T. F. (1997a). Fractional diffusion and Levy stable processes. Phys. Rev. E, 55, 99-106.

West, G. B., Brown, J. H., and Enquist, B. J. (1997b). A general model for the origin of allometric scaling laws in biology. Science 276, 122-126.

Willinger, W. (2000). The Discovery of Self-Similar Traffic. Lecture Notes in Computer Science 1769. Springer. pp 513-527.
Willinger, W., Taquu, M. S., Sherman, R., and Wilson, D. V. (1995). Selfsimilarity through high variability: statistical analysis of Ethernet LAN traffic at the source level. $A C M$ SIGCOMM Comp. Commun. Rev. 25, 100-113.

Wilson, K. G. (1979). Problems in Physics with many scales of length. Sci. Am 241, 158-179.

Wise, M. E. (1981). Spike Interval Distributions for Neurons and Random Walks with Drift to a Fluctuating Threshold. Statistical Distributions in Scientific Work, Vol. 6, eds Taillie et al. (Dordrecht: Reidel Publ. Comp.) 211-231.

Witten, Jr., T.A., and Sander, L. M. (1981) Diffusion-limited aggregation, a kinetic critical phenomenon. Phys. Rev. Lett. 47, 1400-1403.

Womelsdorf, T., Schoffelen, J. M., Oostenveld, R., Singer, W., Desimone, R., and Engel,A. K., and Fries, P. (2007) Modulation of neuronal interactions through neuronal synchronization. Science 316, 1609-1612.

Wornell, G. W. (1993). Wavelet-based representations for the $1 / \mathrm{f}$ family of fractal processes. Proc. IEEE, 81 , 1428-1450.

Xu, Z., Payne, J. R., and Nelson, M. E. (1996). Logarithmic time course of sensory adaptation in electrosensory afferent nerve fibers in a weakly electric fish. J. Neurophysiol. 76 , 2020-2032.

Yamamoto, M., Nakahama, H., Shima, K. Kodama, T., and Mushiake, H. (1986). Markov dependency and spectral analyses on spike counts in mesencephalic reticular neurons during sleep and attentive states. Brain Res. 366, 279-289.

Yeomans, J. M. (1992). Statistical Mechanics of Phase Transitions. Oxford, UK: Oxford Science Publications, Clarendon Press.

Yu, Y., Romero, R., and Lee, T. S. (2005) Preference of sensory neural coding for 1/f signals. Phys. Rev. Lett. 94, 108103.

Yule, G. U. (1925). A mathematical theory of evolution, based on the conclusion of Dr. J. C. Willis. F. R. S. Phil. Trans R. Soc. Lond. B, 213, 21-87.

Zanette, D. H. (2008). Zipf's law and the creation of musical context. arXiv:cs/0406015v1.
Zapperi, S., Lauritsen, K. B., and Stanley, H. E. (1995). Self-organized branching process: mean-field theory for avalanches. Phys. Rev. Lett. 75, 4071-4074.

Zarahn, E., Aguirre, G. K., and D'Esposito, M. (1997). Empirical analyses of BOLD fMRI statistics. Neuroimage 5, 179-197.

Zhang, L. (2006). Quantifying Brain White Matter Structural Changes in Normal Aging Using Fractal Dimension. Doctoral Thesis, Case Western Reserve University. http://etd.ohiolink.edu/sendpdf.cgi / Zhang\% $20 \mathrm{Luduan}$. pdf?acc_num $=$ case 1126213038 .

Zhou, C., Zemanova, L., Zamora-Lopez, G., C. C., and Kurths, J. (2007) Structure-function relationship in complex brain networks expressed by hierarchical synchronization. New J. Phys. 9, 178

Zietsch, B., and Elston, G. N. (2005). Fractal analysis of pyramidal cells in the visual cortex of the Galago (Otolemur Garnetti): regional variation in dendritic branching patterns between visual areas. Fractals 13, 83-90.

Zumofen, G., and Klafter, J. (1993). Scale-invariant motion in intermittent chaotic systems. Phys. Rev. E, 47, 851-863.

Conflict of Interest Statement: The author declare that the research was conducted in the absence of any commercial or financial relationships that could be construed as a potential conflict of interest.

Received: 18 April 2010; paper pending published: 26 May 2010; accepted: 05 June 2010; published online: 06 July 2010.

Citation: Werner G (2010) Fractals in the nervous system: conceptual implications for theoretical neuroscience. Front. Physio. 1:15. doi: 10.3389/fphys.2010.00015

This article was submitted to Frontiers in Fractal Physiology, a specialty of Frontiers in Physiology.

Copyright (c) 2010 Werner. This is an open-access article subject to an exclusive license agreement between the authors and the Frontiers Research Foundation, which permits unrestricted use, distribution, and reproduction in any medium, provided the original authors and source are credited. 